\title{
From big data to diagnosis and prognosis: gene expression signatures in liver hepatocellular carcinoma
}

\author{
Hong Yang ${ }^{1}$, Xin Zhang ${ }^{2}$, Xiao-yong Cai ${ }^{3}$, Dong-yue Wen ${ }^{1}$, Zhi-hua Ye ${ }^{2}$, Liang Liang ${ }^{3}$, Lu Zhang ${ }^{2}$, Han-

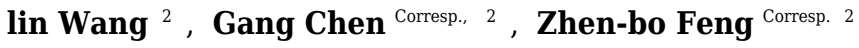 \\ 1 Department of Ultrasonography, First Affiliated Hospital of Guangxi Medical University, Nanning, Guangxi, China \\ 2 Department of Pathology, First Affiliated Hospital of Guangxi Medical University, Nanning, Guangxi, China \\ 3 Department of General Surgery, First Affiliated Hospital of Guangxi Medical University (West Branch), Nanning, Guangxi, China \\ Corresponding Authors: Gang Chen, Zhen-bo Feng \\ Email address: chen_gang_triones@163.com, guanghu1963@126.com
}

Background. Liver hepatocellular carcinoma accounts for the overwhelming majority of primary liver cancers and its belated diagnosis and poor prognosis call for novel biomarkers to be discovered, which, in the era of big data, innovative bioinformatics and computational techniques can prove to be highly helpful in. Methods. Big data aggregated from The Cancer Genome Atlas and Natural Language Processing were integrated to generate differentially expressed genes. Relevant signaling pathways of differentially expressed genes went through Gene Ontology enrichment analysis, Kyoto Encyclopedia of Genes and Genomes and Panther pathway enrichment analysis and protein-protein interaction network. The pathway ranked high in the enrichment analysis was further investigated, and selected genes with top priority were evaluated and assessed in terms of their diagnostic and prognostic values. Results. A list of 389 genes was generated by overlapping genes from The Cancer Genome Atlas and Natural Language Processing. Three pathways demonstrated top priorities, and the one with specific associations with cancers, 'pathways in cancer', was analyzed with its four highlighted genes, namely, BIRC5, E2F1, CCNE1, and CDKN2A, which were validated using Oncomine. The detection pool composed of the four genes presented satisfactory diagnostic power with an outstanding integrated AUC of $0.990(95 \% \mathrm{Cl}: 0.982-0.998$, $P<0.001$, sensitivity: $96.0 \%$, specificity: $96.5 \%)$. BIRC5 ( $P=0.021)$ and CCNE1 $(P=0.027)$ were associated with poor prognosis, while CDKN2A $(P=0.066)$ and E2F1 $(P=0.088)$ demonstrated no statistically significant differences. Discussion. The study illustrates liver hepatocellular carcinoma gene signatures, related pathways and networks from the perspective of big data, featuring the cancer-specific pathway with priority, 'pathways in cancer'. The detection pool of the four highlighted genes, namely BIRC5, E2F1, CCNE1 and CDKN2A, should be further investigated given its high evidence level of diagnosis, whereas the prognostic powers of BIRC5 and CCNE1 are equally attractive and worthy of attention. 


\section{From Big Data to Diagnosis and Prognosis: Gene}

2 Expression Signatures in Liver Hepatocellular 3 Carcinoma

4

5 Hong Yang1\#, Xin Zhang2\#, Xiao-yong Cai3, Dong-yue Wen1, Zhi-hua Ye2, Liang Liang3, Lu

6 Zhang2, Han-lin Wang2, Gang Chen2*, Zhen-bo Feng2*

7 1. Department of Ultrasonography, First Affiliated Hospital of Guangxi Medical University, 6

8 Shuangyong Road, Nanning, Guangxi Zhuang Autonomous Region, P. R. China;

9 2. Department of Pathology, First Affiliated Hospital of Guangxi Medical University, 6

10 Shuangyong Road, Nanning, Guangxi Zhuang Autonomous Region, P. R. China;

11 3. Department of General Surgery, First Affiliated Hospital of Guangxi Medical University (West

12 Branch), 166 Daxue Road East, Nanning, Guangxi Zhuang Autonomous Region, P. R. China;

13 \# Equal contributors as first authors

14 * Contributed equally as corresponding authors; Correspondence shall be addressed to Prof. Gang

15 Chen (chen gang triones@163.com) and Prof. Zhen-bo Feng (guanghu1963@126.com).

16 Emails: Hong Yang: yanghonggx@163.com; Xin Zhang: xinzhdaniel@gmail.com; Xiao-

17 yong Cai: cxy0771@163.com; Dong-yue Wen: ㄴ44653793@qq.com; Zhi-hua Ye:

18 2431618197@qq.com; Liang Liang: 164961885@qq.com; Lu Zhang: 675103122@qq.com; Han-

19 lin Wang: 1164471593@qq.com; Gang Chen: chen_gang_triones@163.com; and Zhen-bo Feng:

20 guanghu1963@126.com. 


\section{Abstract}

23 Background. Liver hepatocellular carcinoma accounts for the overwhelming majority of primary

24 liver cancers and its belated diagnosis and poor prognosis call for novel biomarkers to be discovered, which, in the era of big data, innovative bioinformatics and computational techniques can prove to be highly helpful in.

Methods. Big data aggregated from The Cancer Genome Atlas and Natural Language Processing were integrated to generate differentially expressed genes. Relevant signaling pathways of differentially expressed genes went through Gene Ontology enrichment analysis, Kyoto Encyclopedia of Genes and Genomes and Panther pathway enrichment analysis and proteinprotein interaction network. The pathway ranked high in the enrichment analysis was further investigated, and selected genes with top priority were evaluated and assessed in terms of their diagnostic and prognostic values.

Results. A list of 389 genes was generated by overlapping genes from The Cancer Genome Atlas and Natural Language Processing. Three pathways demonstrated top priorities, and the one with specific associations with cancers, 'pathways in cancer', was analyzed with its four highlighted genes, namely, BIRC5, E2F1, CCNE1 and CDKN2A, which were validated using Oncomine. The detection pool composed of the four genes presented satisfactory diagnostic power with an outstanding integrated AUC of 0.990 (95\%CI: 0.982-0.998, $\mathrm{P}<0.001$, sensitivity: 96.0\%, specificity: 96.5\%). BIRC5 ( $\mathrm{P}=0.021)$ and $\mathrm{CCNE} 1(\mathrm{P}=0.027)$ were associated with poor prognosis,

41 while CDKN2A $(\mathrm{P}=0.066)$ and $\mathrm{E} 2 \mathrm{~F} 1 \quad(\mathrm{P}=0.088)$ demonstrated no statistically significant 42 differences. 
43 Discussion. The study illustrates liver hepatocellular carcinoma gene signatures, related pathways

44 and networks from the perspective of big data, featuring the cancer-specific pathway with priority,

45 'pathways in cancer'. The detection pool of the four highlighted genes, namely BIRC5, E2F1,

46 CCNE1 and CDKN2A, should be further investigated given its high evidence level of diagnosis,

47 whereas the prognostic powers of BIRC5 and CCNE1 are equally attractive and worthy of

48 attention. 


\section{Introduction}

51 Liver cancer ranks the fourth amidst the commonest malignancies globally with its highest incidence in East and South-East Asia together with Northern and Western Africa, taking the second lead among all the cancer-related deaths worldwide (Torre et al. 2015). According to the latest Global Cancer Statistics, a global estimation of 782,500 new incidents of liver cancer and 745,500 deaths occurred during the year 2012, with China alone taking up approximately half of the number (Torre et al. 2015). Liver hepatocellular carcinoma (LIHC), commonly known as 
71 various oncological researches. High-throughput technology, including microarray analysis and

72 RNA sequencing, steals the show, which has now enabled researchers to obtain massive expression data sets, and proven itself to be advantageous and serviceable for identifying novel tumor markers with regard to cancer diagnosis and targeted treatment (Cheng et al. 2013; Horie-Inoue \& Inoue 2013; Xu et al. 2015; Zhang et al. 2015e). The Cancer Genome Atlas (TCGA), a public database begun in 2006, catalogues genetic information and covers 33 types of cancers, which helps facilitate related studies on gene signatures and tumorigenesis mechanisms. To date, only one single paper (Ho et al. 2015) was published with respect to using data from TCGA to investigate gene signatures for LIHC(Li et al. 2014; Lopez-Ayllon et al. 2015; Lu et al. 2014; Wang et al. 2015a; Wang et al. 2015b; Zhang et al. 2015c; Zhang et al. 2015d). As presented in the previous study, Ho, et al. (Ho et al. 2015) employed TCGA whole-transcriptome sequencing data to explore data from 50 paired samples and no other bioinformatics platforms were taken into consideration. Equally attractive is the technology of natural language processing (NLP), which concerns with the interactions between computers and natural languages and has been considered auspicious in the field of human-computer interaction. In the medical profession, researchers regard NLP as one In the study, at first, we combined data from TCGA with genes selected through NLP to analyze the differentially expressed genes (DEGs) in LIHC. Later on, relevant signaling pathways of DEGs 
92 were also investigated using Gene Ontology (GO) enrichment analysis, Kyoto encyclopedia of

93 Genes and Genomes (KEGG) and Panther pathway enrichment analysis and protein-protein 94 interaction (PPI) network. Lastly, the pathway ranked top in KEGG enrichment analysis was

95 further investigated, and selected genes with priority, which might serve as potential biomarkers

96 for the early diagnosis and prognosis prediction, were evaluated and assessed in terms of their

97 diagnostic and prognostic values of several genes (Figure 1). 
99 Materials and Methods

100

101

102

103

104

105

106

107

108

109

110

111

112

113

114

115

116

117

118

119

\section{RNA-seq Data from TCGA Database and DEGs Identification}

The publicly available RNA-seq data of the mRNA level of Liver hepatocellular carcinoma [LIHC] samples were downloaded directly from the TCGA data portal (https://tcgadata.nci.nih.gov/tcga/tcgaHome2.jsp) via bulk download mode [LIHC (cancer type), RNASeqV2 (data type), level 3 (data level), All (preservation) and 1.12.0 (data version)] on January 2, 2017. The data were sequenced based on Illumina Genome Analyzer RNA Sequencing platform. Gene expression data from RNA-Seq-V2 results were quantified through RNA-Seq by ExpectationMaximization (RSEM) (Cheng et al. 2015; Guo et al. 2013) using the "rsem.gene.normalized_results" file type. Extracted data were applied with no further transformation, except by rounding off values to integers. These downloaded data included a total of 369 LIHC samples and 50 non-cancerous liver samples. The DEGs between the LIHC and the non-cancerous liver control samples were identified by using DESeq package [Anders, 2010 \#798] in R. Gene expression comparison was carried out by calculating the level of fold change (FC) in LIHC vs non-cancerous liver tissue. The genes with a $\mathrm{FC}$ value $>2$ or $<0.5$ and with $\mathrm{P}$ value $<0.05$ analyzed with Student's t-test were selected out as DEGs in the current study.

\section{Natural Language Processing}

As previously reported (Zhang et al. 2016), the NLP procedure consisted of searching through electronic records, managing data and statistical analysis. At first, a comprehensive search was conducted in PubMed in order to mine out all the related electronic records, with publishing date ranging from January 1, 1980 to May 25, 2015. The followings are the search strategy employed: 
120 (hepatocellular carcinoma) AND (resistance OR prognosis OR metastasis OR recurrence OR

121 survival OR carcinogenesis OR sorafenib OR bevacizumab) and ('1980/01/01”, [PDAT]:

122 “2015/05/25” [PDAT]). Next, all the pertinent molecules, mostly proteins and genes, were dug out

123 and a list of them was generated afterwards. Gene mention tagging was managed with A

124 Biomedical Named Entity Recognizer (ABNER) (http://pages.cs.wisc.edu/ bsettles/abner/).

125 ABNER also assisted us in conjunction resolution (Settles 2005). Gene name normalization

126 conforms to the standard names in Entrez Database set forth by NCBI (Morgan et al. 2008; Smith

127 et al. 2008). At last comes the statistical analysis. Each gene's frequency of occurrences was

128 calculated individually. The higher a certain gene's frequency appeared to be, the greater chance

129 the gene harbored to be related to LIHC. N was defined as the total number of records in PubMed.

130 Meanwhile, $\mathrm{m}$ and $\mathrm{n}$ represented the occurrence frequencies of genes and LIHC in the PubMed

respectively. K was determined as the frequency of spontaneous co-occurrence of the specific gene

and LIHC under actual circumstances. With hypergeometric distribution, we were able to calculate

and output the probability of occurrence frequency of co-citation greater than $\mathrm{k}$ under total

randomness. The formulae used were as follows:

135

$$
\begin{gathered}
p=1-\sum_{i=0}^{k-1} p(i \mid n, m, N) \\
p(i \mid n, m, N)=\frac{n !(N-n) ! m !(N-m) !}{(n-i) ! i !(n-m) !(N-n-m+i) ! N !}
\end{gathered}
$$

Protein-Protein Interactions Network Construction

Overlaps of DEGs identified from TCGA and genes obtained from NLP were calculated using the

COUNTIF function in Microsoft Excel 2013. STRING (http://string-db.org/), Search Tool for the 
140 Retrieval of Interacting Genes/Proteins, is a bioinformatics platform and web resource of known

141 and predicted PPI network. The latest version STRING 10.0 was employed to construct PPI

142 network of proteins encoded by the extracted DEGs with the cut-off criterion of combined score

$143>0.7$. The PPI data were downloaded from STRING database and a map of the complete PPI

144 network was drawn. The interactions of proteins derived from four sources, i.e. 1) literature-

145 reported protein interactions, 2) high-throughput experiments, 3) genome analysis and prediction

146 and 4) co-expression studies. Next, the hub genes, namely highly connected genes in the network,

147 were extracted from PPI data using the R Project for Statistical Computing (https://www.r-

148 project.org/), an open access software environment for statistical computation and graphics.

149 Moreover, STRING was in charge of both the screening of top hub genes and the visualization of

150 network. The protein product of a certain gene acts as a node in the PPI network, and the

151 connectivity degree evinces the number of interplayed proteins. A node with high connectivity

152 degrees is considered as a hub node. Hub proteins were obtained by means of analyzing the

153 connectivity degrees of the nodes in PPI networks.

154 Functional and Signaling Pathway Analyses

155 A set of condition-specific genes from the overlaps of TCGA and NLP further underwent the 156 functional and signaling pathway analyses. The functional and signaling pathway analyses of the 157 selected DEGs was performed on a public database platform, the Database for Annotation, 158 Visualization and Integrated Discovery (DAVID) (https://david.ncifcrf.gov/), which could provide 159 a functional interpretation of massive gene lists deriving from genomic studies. The analyses 160 included gene ontology (GO) function analysis (http://www.geneontology.org/), Kyoto 
161 Encyclopedia of Genes, Genomes (KEGG) (http://www.genome.jp/kegg/) analysis and panther

162 pathway analysis. GO function analysis categorized selected genes into groups in accordance with

163 three independent classification standards, namely molecular function (MF), cellular component

164 (CC), and biological process (BP). A Benjamini P-value of $<0.05$ was used in the above pathway

165 enrichment analyses. The results were visualized into three GO-maps via Cytoscape v3.4.0

166 (http://cytoscape.org/). Subsequently, the highly relevant pathway with the top priority was

167 selected for further evaluation, and the most significantly aberrantly expressed genes were

168 examined for their prospective diagnostic and prognostic values. The aberrantly expressed genes

169 selected were bioinformatically validated using Oncomine Research Edition [Rhodes, 2004 \#799]

170 (https://www.oncomine.org/).

171 Statistical Analysis

172 SPSS 22.0 was used for statistical analysis. All the data were presented as mean \pm standard

173 deviation of mean. The receiver operating characteristic (ROC) curve was drawn to identify the

174 diagnostic significance of genes separately. The logistic regression contributed to evaluating the

integrated diagnostic value of the combined pool of the top four genes in LIHC. The standards for

assessing the area under the curve (AUC) in ROC were as follows: $0.5-0.7$ (poor evidence for

177 diagnosis); 0.7-0.9 (moderate evidence for diagnosis); 0.9-1.0 (high evidence for diagnosis).

Significance of difference between LIHC and para-LIHC non-cancerous liver tissues was analyzed

by Student's t-test. The scatter plot to exhibit the level of gene expression was demonstrated by

GraphPad Prism 5.0. Overall survival (OS) was estimated by the Kaplan-Meier method, and the

log-rank test was conducted to compare survival curves. Kaplan-Meier survival curve was drawn 
182 to evaluate the associations between DEGs expressions and survival rates of 369 patients with 183 LIHC. P value less than 0.05 indicated statistical significance. 
185 Results

186 Natural Language Processing

187 A series of 64,577 LIHC-related electronic records of titles and abstracts altogether was retrieved

188 from PubMed. Later on, statistical analysis highlighted a panel of 1800 LIHC-related genes.

\section{Overlaps between DEGs from TCGA and LIHC-related genes from NLP}

A total of 3278 DEGs were achieved from TCGA dataset between LIHC patients and non-LIHC

liver controls in accordance with the criteria described in the 'materials and methods' section.

Meanwhile, 1800 LIHC-related genes from NLP procedure proceeded (Zhang et al. 2016). The

integration witnessed a set of 389 genes by overlapping DEGs from TCGA and LIHC-related genes from NLP.

\section{PPI Network Analysis}

The PPI network was constructed with STRING in an attempt to systemically analyze the functions

of DEGs. The map of complete PPI network has been made available in Supplementary Figure 1.

And a core sub-network of 22 genes was selected for further analyses, whose connectivity degrees were more than 20 as presented in Figure 2.

\section{Functional and Signaling Pathway Analyses}

201

GO analysis classified 389 DEGs into three GO categories, namely 353 DEGs along with 613

202

pathways in BP, 304 DEGs together with 67 pathways in CC and 333 DEGS with 93 pathways in

203

MF. In BP, the top three processes which DEGs actively participated in were response to organic substance (GO: $0010033, \mathrm{P}=1.93 \times 10^{-22}$ ), regulation of phosphorylation (GO: 0042325,

$\mathrm{P}=2.34 \times 10^{-19}$ ) and regulation of phosphorus metabolic process (GO: 0051174, $\left.\mathrm{P}=1.36 \times 10^{-18}\right)$. 
206 Meanwhile, in CC, extracellular region part (GO: 0044421, $\mathrm{P}=1.81 \times 10^{-14}$ ), extracellular space 207 (GO: 0005615, $\mathrm{P}=7.67 \times 10^{-13}$ ) and spindle ( $\mathrm{GO}: 0005819, \mathrm{P}=4.67 \times 10^{-12}$ ) were considered the top 208 three. In MF, the top three functional items were cadmium ion binding (GO: $0046870, \mathrm{P}=7.81 \times 10^{-}$ $209^{5}$ ), platelet-derived growth factor binding (GO: 0048407, $\left.\mathrm{P}=1.20 \times 10^{-4}\right)$ and kinase binding (GO: $\left.2100019900, \mathrm{P}=2.05 \times 10^{-4}\right)$. In addition, the gene expression profile graph from the Global Cancer 211 Map was also processed in Gene Set Enrichment Analysis (GSEA) and the Molecular Signatures 212 Database (MSigDB, http://software.broadinstitute.org/gsea/datasets.jsp). GSEA is a 213 computational solution that decides whether an a priori defined set of genes demonstrates 214 statistically significant, concordant differences between two biological environments, while 215 MSigDB is a collected affiliation of annotated gene sets for GSEA software. Three visualized GO216 maps (BP, CC, MF) by Cytoscape v3.4.0 and GSEA were rendered in, Figure 3, Figure 4, Figure 217 5, and Supplementary Figure 2. We identified the expression of significantly altered genes among 218 the DEGs, as well as their relative tendencies of expression in the defined functional groups (Figure $2196)$.

KEGG pathway enrichment analysis demonstrated the significant enrichments of DEGs in 10 items (Figure 5). The three prominent pathways with top significance were cell cycle (hsa04110, $\left.\mathrm{P}=2.58 \times 10^{-9}\right)$, pathways in cancer $\left(\mathrm{hsa} 05200, \mathrm{P}=5.40 \times 10^{-6}\right)$ and Toll-like receptor signaling 223 pathway (hsa $\left.04620, \mathrm{P}=6.31 \times 10^{-5}\right)$.

224 Panther pathway enrichment analysis identified three pathways with top significance, i.e. p53 225 pathway ( $\mathrm{P} 00059, \mathrm{P}=0.001)$, Toll receptor signaling pathway $(\mathrm{P} 00054, \mathrm{P}=0.005)$ and blood 226 coagulation $(\mathrm{P} 00011, \mathrm{P}=0.007)$. 
227 Compared with other databases, KEGG is an ontology database illustrating functional hierarchic

228 domains of various biological entities, including molecules, cells, organisms, drugs and diseases,

229 along with relationships among them. Thus, we decided to select the cancers-specific pathway

230 with prominent significance from KEGG analysis, 'pathways in cancer' with 30 genes included,

231 to further analyze the diagnostic values of the target genes. The positions of 30 genes in "pathways

232 in cancer" were shown in Figure 7.

233 Diagnostic Values of Highlighted DEGs

234 Thirty significant aberrant genes involved in the highlighted pathway, pathways in cancer, which

235 is considered the most cancer-related and ranked top in KEGG analysis, were assessed for

236 diagnostic values by means of ROC curves. There were 12 genes with AUCs ranging from 0.9-

$2371.0,15$ genes demonstrating AUCs between 0.7-0.9, and three genes presenting AUCs from 0.5 to

238 0.7. Delightfully, 12 genes harbored AUCs exceeding 0.9. The AUCs of the top four genes, i.e.

239 BIRC5, E2F1, CCNE1 and CDKN2A, were 0.974 (95\%CI: 0.960-0.989, P<0.001), 0.964 (95\%CI:

240

0.942-0.986, $\mathrm{P}<0.001), 0.945(95 \% \mathrm{CI}: 0.919-0.971, \mathrm{P}<0.001)$ and 0,939 (95\%CI: 0.915-0.963,

$\mathrm{P}<0.001$ ), respectively. Four of them have been all marked red in Figure 8. Genes with AUCs $>0.8$

242 were displayed in Figure 9.

243 For the purpose of validation, BIRC5, ECF1, CCNE1 and CDKN2A were searched in Oncomine

244 Research Edition [Rhodes, 2004 \#799] (https://www.oncomine.org) filtered with the Cancer Type

245 of Liver Cancer. Oncomine is an online platform gathering cancer expression datasets and

246 providing data mining services, which currently includes 715 datasets and 86,733 samples as of

247 Jan 15, 2017 [Rhodes, 2004 \#799]. All these four genes were included in different datasets. Highly 
248 and reasonably consistent with the results from TCGA, all BIRC5 (Supplementary Figure 3A, 3B

249 and 3C), ECF1 (Supplementary Figure 3D), CCNE1 (Supplementary Figure 3E) and CDKN2A

250 (Supplementary Figure 3F and 3G) showed evidently over-expressed pattern in LIHC tissues.

251 Gene expressions between LIHC and para-LIHC non-cancerous tissues were also compared. The expressions of top four genes in LIHC tissues were higher than those in the corresponding adjacent non-cancerous tissues, and the results were as follows: $332.95 \pm 530.89$ vs $11.51 \pm 11.07(\mathrm{t}=11.612$,

$\mathrm{P}<0.001), 616.43 \pm 765.05$ vs $41.45 \pm 48.14(\mathrm{t}=14.230, \mathrm{P}<0.001), 239.49 \pm 1030.09$ vs $10.09 \pm 8.23$

$(\mathrm{t}=4.277, \mathrm{P}<0.001)$ and $427.99 \pm 529.58$ vs $21.66 \pm 23.55(\mathrm{t}=14.632, \mathrm{P}<0.001)$, respectively (Figure

10). Moreover, the four genes with the highest AUCs were combined as a pool to distinguish LIHC

tissues from the non-tumor ones. The integrated AUC reached a satisfying point of $0.990(95 \% \mathrm{CI}$ :

0.982-0.998, $\mathrm{P}<0.001$ ), which indicated its remarkable significance for LIHC diagnosis. The

sensitivity and specificity of the pool were $96.0 \%$ and $96.5 \%$, respectively.

Prognostic Values of Highlighted DEGs

In order to evaluate the prognostic values of highlighted DEGs, we further investigated the associations of the top four DEGs with overall survival (OS) of patients by Kaplan-Meier and logrank analysis. Among all the 369 patients involved, 368 had complete followed-up data.

Determined by ROC analyses, the cut-off values for the highlighted genes were 41.76 for BIRC5,

17.85 for CCNE1, 81.90 for CDKN2A, and 101.37 for E2F1, respectively. Patients with lower

level of BIRC5 expression $(n=59)$ expected longer survival time $(67.10 \pm 6.30,95 \% \mathrm{CI}$ : 54.74 -

79.44) than those with higher level of BIRC5 expression $(n=309)(60.67 \pm 4.04,95 \%$ CI: 52.75 -

68.58, $\mathrm{P}=0.021)$. Patients with lower CCNE1 expression $(\mathrm{n}=47)$ were inclined to demonstrate 
269 longer survival $(73.34 \pm 7.40,95 \% \mathrm{CI}: 58.83-87.84)$ than patients with higher CCNE1 expression $270(\mathrm{n}=321)(59.91 \pm 4.03,95 \% \mathrm{CI}: 52.01-67.80, \mathrm{P}=0.027)$. However, no statistically significant 271 differences between low and high expression groups of CDKN2A $(\mathrm{P}=0.066)$ and $\mathrm{E} 2 \mathrm{~F} 1(\mathrm{P}=0.088)$ 272 were observed in terms of survival (Figure 11 A-D). 


\section{Discussion}

275 Genes and proteins usually function via complex networks and have the ability to affect the operations of biological systems collaboratively. Furthermore, the impact of multi-gene interactions on cellular functions through signaling pathways has been proved to be more influential than that of a single gene. Up to now, the pathogenesis of LIHC has been widely investigated and the consensus emerged that multi-gene interactions contribute to the carcinogenesis and progression of LIHC (Li et al. 2014; Lopez-Ayllon et al. 2015; Lu et al. 2014;

Wang et al. 2015a; Wang et al. 2015b; Zhang et al. 2015c; Zhang et al. 2015d). In the present study, multi-gene analyses with various bioinformatics techniques and platforms, such as NLP and TCGA, were performed to identify the aberrantly expressed genes in LIHC. The condition-specific genes were obtained from the overlapping of TCGA and NLP, which further proceeded with functional and pathways enrichment analysis so as to render prospective biomarkers for LIHC diagnosis and survival prediction and to elucidate the potential mechanism of the DEGs in LIHC. Antecedently, articles published by Jin, et al. (Jin et al. 2015a), Ho, et al. (Ho et al. 2015) and Shangguan, et al. (Shangguan et al. 2015) investigated the deregulated genes and related signaling pathways in LIHC by bioinformatics techniques. As compared with the previous studies, the current one is the first to analyze the DEGs in LIHC by the winning combination of TCGA database and NLP analysis. It should be highlighted that the sample cohort in this present analysis consisted of 369 LIHC and 50 para-LIHC non-cancerous liver tissues, outweighing any formerly reported studies, that is, 117 tissue samples (54 LIHC cases and 63 controls) in the study of Jin, et 
286 LIHC and 39 non-cancerous liver tissues explored by Shangguan, et al (Shangguan et al.

296

297

298

299

300

301

302

303

304

305

306

307

308

309

310

311

312

313

314

315

2015). More importantly, the current study evaluated the diagnostic and prognostic values of the

featured hub genes in LIHC by means of ROC curves and survival analysis. Given the above, the

study might provide more profound insights than preceding researches which ignored the application in diagnosis and prognosis but solely focused on the hub genes, dysregulated pathways and relevant molecular mechanisms.

Novel signaling pathways analysis enables us to learn more about the pathogenesis, biological

processes and the key pathogenic genes of cancers. For instance, Ho, et al.(Ho et al. 2015) employed TCGA whole-transcriptome sequencing data and discovered the significantly enriched

KEGG pathways of cell cycle and p53 signaling, which matches our results in the present one with

DEGs from TCGA and supports the credibility and reliability of the current research. Other studies employed Gene Expression Omnibus (GEO) data to perform KEGG pathways enrichment analysis but their results did not coincide with ours (Jin et al. 2015a; Li et al. 2016). Such findings might be related to the methods and sample sizes adopted. We not only used LIHC data from TCGA which harbors a large number of samples, but also combined them with the genes generated by NLP, both of which make our research stand out among all the others.

The KEGG Pathway Database is believed to be one of the most comprehensive knowledge bases to understand organisms as molecular systems in the development of tumor-genesis, proliferation, metastasis and apoptosis. Among the signaling pathways presented, pathways in cancer $(\mathrm{P}=5.40 \mathrm{E}-$ 06, FDR=0.06) ranked top among the list of KEGG pathways released by DAVID and is considered specifically associated with cancers, which implied that pathways in cancer might be 
actively involved in the oncogenesis and development of LIHC and suggested that its target genes

317 could be potential markers for diagnosis and prognosis.

318 Alpha-fetoprotein (AFP) is considered a vital and mature biomarker in the diagnosis of liver

319 cancer, especially in the case of LIHC. However, the sensitivity of AFP is subject to the size and

320 stage of tumor, which makes combined detection of multiple biomarkers relatively superior and

321 more convincing. Thus, we decided to concentrate on the featured pathway, i.e. pathways in

322 cancer, as well as its related genes.

323 Of the 389 DEGs identified in the RNA-seq profiles of TCGA LIHC database, 30 genes were

found to be the most enriched in pathways in cancer, which indicated an increased possibility of

genes functioning in the factual development and progression of LIHC. Their diagnostic values

were evaluated for by ROC curves, where 12 genes harbored high diagnostic value by AUCs

exceeding 0.9 and another 10 genes demonstrated moderate diagnostic importance with AUCs

ranging from 0.8 to 0.9 (Figure 9). Four special genes with the highest AUCs, namely BIRC5,

E2F1, CCNE1 and CDKN2A, were validated to be over-expressed in LIHC tissues using

Oncomine [Rhodes, 2004 \#799]. The Oncomine validation not only boosts the feasibility of using

the four-gene pool as a potential diagnostic strategy but also prove the powerful validity of the

bioinformatics methods used. We consider that the bioinformatics validation adds much to the

significance and credibility of the study. With the four genes validated, we became curious about

the potential to diagnose LIHC with the assistance of a pool formed by the four genes. The four-

gene pool excelled with an outstanding integrated AUC of $0.990(95 \% \mathrm{CI}: 0.982-0.998, \mathrm{P}<0.001$, 
337 Meanwhile, the results of ROC curves furnished us with more accurate information to distinguish

338 the survival differences between high and low expression groups of certain genes. Overall, patients

339 with low BIRC5 expression $(n=59)$ showed longer survival time $(67.10 \pm 6.30,95 \%$ CI: 54.74-

340 79.44) than those with high BIRC5 expression $(n=309)(60.67 \pm 4.04,95 \%$ CI: 52.75-68.58,

$341 \mathrm{P}=0.021$ ). Similar results could be witnessed in the case of CCNE1, where patients with low

342 CCNE1 expression $(n=47)$ demonstrated longer survival $(73.34 \pm 7.40,95 \%$ CI: $58.83-87.84)$ than

343 patients with high CCNE1 expression $(n=321)(59.91 \pm 4.03,95 \% C I$ : 52.01-67.80, $\mathrm{P}=0.027)$.

344 Nonetheless, CDKN2A $(\mathrm{P}=0.066)$ or $\mathrm{E} 2 \mathrm{~F} 1(\mathrm{P}=0.088)$ displayed no statistically significant

345 differences between low and high expression groups.

346 It is strikingly noteworthy that the four-gene detention pool has been proved to be auspicious for

347 LIHC diagnosis, which consisted of BIRC5, E2F1, CCNE1 and CDKN2A. In the meanwhile, the

348 roles that BIRC5 and CCNE1 might play in the prognosis of LIHC should not be ignored. Earlier

349 studies regarding the four genes might help account for the possible molecular mechanisms.

BIRC5 (Baculoviral IAP repeat containing 5), located in 17q25, belongs to the inhibitor of

apoptosis (IAP) gene family, which encodes apoptosis-preventing proteins via negative regulation.

352 Biologically, BIRC5 plays a crucial role in malignancy through inhibiting cell apoptosis,

enhancing cellular proliferation and promoting angiogenesis(Ryan et al. 2009). Yu, et al. found

that the expression of BIRC5 was increased in LIHC tissues as compared to those adjacent non-

tumor samples and non-cancerous liver tissues (Yang et al. 2011). Several articles demonstrated 
358 2007). Recently, the investigation of the role of non-coding RNAs in cancer has attracted

359 increasing attention. Chen, et al.(Chen et al. 2015) illustrated that miR-153 enhanced BIRC5

360 expression in LIHC cell lines and Wei, et al. (Wei et al. 2013) verified by Western blot assay that

361 miR-203 could target survivin to promote the progression of LIHC. Moreover, hepatitis B virus X

362 protein combined with BIRC5 could accelerate carcinogenesis of LIHC by modulating miR-520b

363 and hepatitis B X-interacting protein (Baginski 1969). Nonetheless, the correlations between long

364 noncoding RNAs (lncRNAs) and BIRC5 were investigated only in lung cancer and prostatic

365 cancer (Bialkowska-Hobrzanska et al. 2006; Xia et al. 2015). Thus, it is necessary to clarify the

366 relationships between lncRNAs and BIRC5 and their functions in the pathogenesis of LIHC.

367 E2F transcription factor 1, also known as E2F1, has been widely studied in the generation and

progression of several cancers. In liver cancer, Lu, et al. (Lu et al. 2016) examined the expression

of E2F1 in 143 LIHC samples using tissue arrays and observed that strong positive staining of

E2F1 took place in $84.62 \%$ of LIHC tissues through immunohistochemical (IHC) staining.

Moreover, Wang, et al.(Wang et al. 2016) reported that E2F1 is an important downstream gene of

ISX in hepatoma progression. Recently, studies of E2F1 have uncovered its critical roles in the

control of transcription, proliferation and apoptosis(DeGregori \& Johnson 2006; Muller et al.

2001; O'Donnell et al. 2005), and accumulating evidence showed that E2F1, a fundamental

biological regulator with the ability to activate transcription in downstream genes, was also

involved in the regulation of other molecules, such as miRNAs and lncRNAs. For instance,

Kathryn, et al. (O'Donnell et al. 2005) elaborated that E2F1 transcription was simultaneously 
379 et al. 2008) unveiled the establishment of a negative feedback loop by the interaction of E2F1 and 380 miR-106b-25 cluster, which might influence the development of TGF $\beta$ resistance in gastric cancer.

promotes or facilitates cell proliferation and cell cycle in gastric cancer. The functions of E2F1 as

a target for other molecules have also been extensively reported, such as miR-106b in bladder cancer(Jin et al. 2015b), miR-136 in glioma cells(Chen et al. 2014), miR-331-3p in gastric cancer(Guo et al. 2010) and so forth.

Cyclin E1 (CCNE1), a cardinal regulator of the G1/S cell-cycle transition and member of cyclins family, was upregulated in LIHC(Peng et al. 1998). The increased expression of CCNE1 might shorten the tumor cell cycle phase, speech up cell proliferation, and be closely involved in LIHC aggressiveness according to the published literatures. For example, Zhou, et al.(Zhou et al. 2003) established that CCNE1 plays a cooperative role in LIHC tumorigenesis, differentiation, invasiveness and metastasis. $\mathrm{Li}$, et al.(Li et al. 2003), used RNA interference to target CCNE1overexpressing in LIHC, and found that CCNE1, overexpressed in $70 \%$ of LIHCs, might serve as a novel therapeutic target. CCNE1, as a spotlighted target gene of micro-RNA, has already been found to be actively involved in LIHC (Zhang et al. 2014). All the above indicate that CCNE1 is a crucial gene involved in the development and progression of LIHC and has the potential to be an effective biomarker for diagnosis and prognosis of LIHC.

CDKN2A (p16INK4a), located on chromosome 9p21, is an important tumor suppressor and DNA repair gene and functions as a major negative regulator of critical tumor pathways(Song et al. 2013). CDKN2A encodes p16 protein (p16) that competitively binds to cyclin-dependent kinase 4 
400

401

402

403

404

405

406

407

408

409

410

411

412

413

414

415

416

417

418

419

420

protein (Cdk4), and therefore inhibits the interaction of Cdk4 and cyclin D1 (Sherr 1996). Liggett

and Sidransky (Liggett \& Sidransky 1998) believed that the p16/cyclin D/CDK/pRB pathway is

prominent in many epithelial malignancies. Inactivation of p16INK4a induced by aberrant

hypermethylation is implicated in the process of carcinogenesis of most common human cancers

and associated with poor prognosis (Sharpless 2005). It has been reported that the methylation of

CDKN2A gene is connected to the incidence of LIHC (Biden et al. 1997). Also, the present study

indicated that CDKN2A expression in LIHC tissues was significantly increased as compared to

para-LIHC non-tumor tissues. Furthermore, Lan, et al. (Lan et al. 2011) discovered that hsalet-7g

might exercise its inhibitory ability via up-regulating p16INK4A and play a considerably

significant part in inhibiting LIHC tumorigenesis and progression.

Despite the demonstration of multiple significantly aberrant genes in LIHC, there still existed

several limitations in the present study. First all of, the results were based solely on the sequencing

data in TCGA database without further in vivo and in vitro confirmation. Moreover, the solely

used RNA-seq technology in TGCA confined the legitimacy of the study, which requires further

validation. Lastly, the samples of LIHC were mainly collected in the western world, which might

fail to represent the worldwide picture of gene signatures in LIHC. Further investigations of DEGs

in LIHC concentrating on Asian and African countries might help mend the issue.

In summary, the study investigated LIHC gene expression signatures, related pathways and networks by overlapping data from TCGA and NLP. One particular pathway, 'pathways in cancer', stood out due to its massive importance. Furthermore, four key genes highly involved in 'pathways in cancer', BIRC5, E2F1, CCNE1 and CDKN2A, were bioinformatically validated and 
421 selected out for further diagnostic and prognostic tests. The detection pool formed by the four 422 genes rendered ideal values in term of diagnosing LIHC. Meanwhile, BIRC5 and CCNE1 can also 423 be considered promising in the field of LIHC prognosis. In the era of big data, we now can gather 424 worldwide gene expression data from multiple databases. A case in point is TCGA, which helps 425 the present research to gain exclusive access to an unprecedentedly massive sample size of LIHC 426 patients along with the expression data. Based on that, the diagnostic and prognostic tests are 427 deemed even more creditable and convincing. Forthcoming research can target the novel non428 invasive detection methods using serum to diagnose LIHC and predict its prognosis on the selected 429 genes, whose pool has been proved effective in the present study. Still, relevant molecular 430 mechanisms in relation to the mentioned genes, pathways, and networks need further investigation 431 and validation. 


\section{Conclusion}

434 The study has not only illustrated gene expression signatures of LIHC and related regulatory

435 pathways and networks from the perspective of big data, featuring the cancers-associated pathway

436 with top priority, 'pathways in cancer', but also analyzed the diagnostic and prognostic values of

437 four highlighted genes, namely BIRC5, E2F1, CCNE1 and CDKN2A, which were validated using

438 Oncomine. It is advisable that a detection pool of the four genes should be further investigated

439 owing to its high evidence level of diagnosis, whereas we believe that the prognostic powers of

440 BIRC5 and CCNE1 are equally attractive and thus worthy of attention. 


\section{References}

443

444

445

446

447

448

449

450

451

452

453

454

455

456

457

458

459

460

461

462

463

464

465

466

467

468

469

470

471

472

473

474

475

476

477

478

479

480

481
Augello C, Caruso L, Maggioni M, Donadon M, Montorsi M, Santambrogio R, Torzilli G, Vaira V, Pellegrini C, Roncalli $\mathrm{M}$, Coggi G, and Bosari S. 2009. Inhibitors of apoptosis proteins (IAPs) expression and their prognostic significance in hepatocellular carcinoma. BMC Cancer 9:125. 10.1186/1471-2407-9-125

Baginski S. 1969. [In memoriam of Associate Professor Jan Kruszynski, M]. Folia Morphol (Warsz) 28:101-102.

Bialkowska-Hobrzanska H, Driman DK, Fletcher R, Harry V, and Razvi H. 2006. Expression of human telomerase reverse transcriptase, Survivin, DD3 and PCGEM1 messenger RNA in archival prostate carcinoma tissue. Can J Urol 13:2967-2974.

Biden K, Young J, Buttenshaw R, Searle J, Cooksley G, Xu DB, and Leggett B. 1997. Frequency of mutation and deletion of the tumor suppressor gene CDKN2A (MTS1/p16) in hepatocellular carcinoma from an Australian population. Hepatology 25:593-597. 10.1002/hep.510250317

Buendia MA. 2000. Genetics of hepatocellular carcinoma. Semin Cancer Biol 10:185-200. 10.1006/scbi.2000.0319

Bupathi M, Kaseb A, and Janku F. 2014. Angiopoietin 2 as a therapeutic target in hepatocellular carcinoma treatment: current perspectives. Onco Targets Ther 7:1927-1932. 10.2147/OTT.S46457

Chen W, Yang Y, Chen B, Lu P, Zhan L, Yu Q, Cao K, and Li Q. 2014. MiR-136 targets E2F1 to reverse cisplatin chemosensitivity in glioma cells. J Neurooncol 120:43-53. 10.1007/s11060-014-1535-x

Chen Y, Feng F, Gao X, Wang C, Sun H, Zhang C, Zeng Z, Lu Y, An L, Qu J, Wang F, and Yang Y. 2015. MiRNA153 Reduces Effects of Chemotherapeutic Agents or Small Molecular Kinase Inhibitor in LIHC Cells. Curr Cancer Drug Targets 15:176-187.

Cheng H, Fertig EJ, Ozawa H, Hatakeyama H, Howard JD, Perez J, Considine M, Thakar M, Ranaweera R, Krigsfeld G, and Chung CH. 2015. Decreased SMAD4 expression is associated with induction of epithelial-tomesenchymal transition and cetuximab resistance in head and neck squamous cell carcinoma. Cancer Biol Ther 16:1252-1258. 10.1080/15384047.2015.1056418

Cheng WC, Kingsbury TJ, Wheelan SJ, and Civin Cl. 2013. A simple high-throughput technology enables gain-offunction screening of human microRNAs. Biotechniques 54:77-86. 10.2144/000113991

Chun JM, Kwon HJ, Sohn J, Kim SG, Park JY, Bae HI, Yun YK, and Hwang YJ. 2011. Prognostic factors after early recurrence in patients who underwent curative resection for hepatocellular carcinoma. I Surg Oncol 103:148-151. 10.1002/jso.21786

Dai J, Yang L, Wang J, Xiao Y, and Ruan Q. 2015. Prognostic Value of FOXM1 in Patients with Malignant Solid Tumor: A Meta-Analysis and System Review. Dis Markers 2015:352478. 10.1155/2015/352478

DeGregori J, and Johnson DG. 2006. Distinct and Overlapping Roles for E2F Family Members in Transcription, Proliferation and Apoptosis. Curr Mol Med 6:739-748.

Dhanasekaran R, Limaye A, and Cabrera R. 2012. Hepatocellular carcinoma: current trends in worldwide epidemiology, risk factors, diagnosis, and therapeutics. Hepat Med 4:19-37. 10.2147/HMER.S16316

Guo X, Guo L, Ji J, Zhang J, Zhang J, Chen X, Cai Q, Li J, Gu Q, Liu B, Zhu Z, and Yu Y. 2010. miRNA-331-3p directly targets E2F1 and induces growth arrest in human gastric cancer. Biochem Biophys Res Commun 398:1-6. 10.1016/j.bbrc.2010.05.082

Guo Y, Sheng Q, Li J, Ye F, Samuels DC, and Shyr Y. 2013. Large scale comparison of gene expression levels by microarrays and RNAseq using TCGA data. PLoS One 8:e71462. 10.1371/journal.pone.0071462

He R, Yang L, Lin X, Chen X, Lin X, Wei F, Liang X, Luo Y, Wu Y, Gan T, Dang Y, and Chen G. 2015. MiR-30a-5p suppresses 
482

483

484

485

486

487

488

489

490

491

492

493

494

495

496

497

498

499

500

501

502

503

504

505

506

507

508

509

510

511

512

513

514

515

516

517

518

519

520

521

522 cell growth and enhances apoptosis of hepatocellular carcinoma cells via targeting AEG-1. Int J Clin Exp Pathol 8:15632-15641.

Ho DW, Kai AK, and Ng IO. 2015. TCGA whole-transcriptome sequencing data reveals significantly dysregulated genes and signaling pathways in hepatocellular carcinoma. Front Med 9:322-330. 10.1007/s11684-015-0408-9

Horie-Inoue K, and Inoue S. 2013. Genome-wide integrated analyses of androgen receptor signaling in prostate cancer based on high-throughput technology. Curr Drug Targets 14:472-480.

Jin B, Wang W, Du G, Huang GZ, Han LT, Tang ZY, Fan DG, Li J, and Zhang SZ. 2015a. Identifying hub genes and dysregulated pathways in hepatocellular carcinoma. Eur Rev Med Pharmacol Sci 19:592-601.

Jin N, Jin X, Gu X, Na W, Zhang M, and Zhao R. 2015b. Screening biomarkers of bladder cancer using combined miRNA and mRNA microarray analysis. Mol Med Rep 12:3170-3176. 10.3892/mmr.2015.3739

Lan FF, Wang H, Chen YC, Chan CY, Ng SS, Li K, Xie D, He ML, Lin MC, and Kung HF. 2011. Hsa-let-7g inhibits proliferation of hepatocellular carcinoma cells by downregulation of c-Myc and upregulation of p16(INK4A). Int J Cancer 128:319-331. 10.1002/ijc.25336

Li J, Huang Z, and Wei L. 2016. Bioinformatics analysis of the gene expression profile of hepatocellular carcinoma: preliminary results. Contemp Oncol (Pozn) 20:20-27. 10.5114/wo.2016.58497

Li K, Lin SY, Brunicardi FC, and Seu P. 2003. Use of RNA interference to target cyclin E-overexpressing hepatocellular carcinoma. Cancer Res 63:3593-3597.

Li MX, Bi XY, Li ZY, Huang Z, Han Y, Zhou JG, Zhao JJ, Zhang YF, Zhao H, and Cai JQ. 2015. Prognostic Role of Glasgow Prognostic Score in Patients With Hepatocellular Carcinoma: A Systematic Review and Meta-Analysis. Medicine (Baltimore) 94:e2133. 10.1097/MD.0000000000002133

Li SC, Tachiki LM, Kabeer MH, Dethlefs BA, Anthony MJ, and Loudon WG. 2014. Cancer genomic research at the crossroads: realizing the changing genetic landscape as intratumoral spatial and temporal heterogeneity becomes a confounding factor. Cancer Cell Int 14:115. 10.1186/s12935-014-0115-7

Liggett WH, Jr., and Sidransky D. 1998. Role of the p16 tumor suppressor gene in cancer. J Clin Oncol 16:1197-1206.

Liu JL, Zhang XJ, Zhang Z, Zhang AH, Wang W, and Dong JH. 2013. Meta-analysis: prognostic value of survivin in patients with hepatocellular carcinoma. PLoS One 8:e83350. 10.1371/journal.pone.0083350

Lopez-Ayllon BD, de Castro-Carpeno J, Rodriguez C, Pernia O, Ibanez de Caceres I, Belda-Iniesta C, Perona R, and Sastre L. 2015. Biomarkers of erlotinib response in non-small cell lung cancer tumors that do not harbor the more common epidermal growth factor receptor mutations. Int J Clin Exp Pathol 8:2888-2898.

Lu X, Ye K, Zou K, and Chen J. 2014. Identification of copy number variation-driven genes for liver cancer via bioinformatics analysis. Oncol Rep 32:1845-1852. 10.3892/or.2014.3425

Lu Z, Xiao Z, Liu F, Cui M, Li W, Yang Z, Li J, Ye L, and Zhang X. 2016. Long non-coding RNA HULC promotes tumor angiogenesis in liver cancer by up-regulating sphingosine kinase 1 (SPHK1). Oncotarget 7:241-254. 10.18632/oncotarget.6280

Morgan AA, Lu Z, Wang X, Cohen AM, Fluck J, Ruch P, Divoli A, Fundel K, Leaman R, Hakenberg J, Sun C, Liu HH, Torres R, Krauthammer M, Lau WW, Liu H, Hsu CN, Schuemie M, Cohen KB, and Hirschman L. 2008. Overview of BioCreative II gene normalization. Genome Biol 9 Suppl 2:S3. 10.1186/gb-2008-9-s2-s3

Muller H, Bracken AP, Vernell R, Moroni MC, Christians F, Grassilli E, Prosperini E, Vigo E, Oliner JD, and Helin K. 2001. E2Fs regulate the expression of genes involved in differentiation, development, proliferation, and apoptosis. Genes Dev 15:267-285. 10.1101/gad.864201

O'Donnell KA, Wentzel EA, Zeller KI, Dang CV, and Mendell JT. 2005. c-Myc-regulated microRNAs modulate E2F1 
expression. Nature 435:839-843. 10.1038/nature03677

Pan L, Huang S, He R, Rong M, Dang Y, and Chen G. 2014. Decreased expression and clinical significance of miR-148a in hepatocellular carcinoma tissues. Eur J Med Res 19:68. 10.1186/s40001-014-0068-2

Peng SY, Chou SP, and Hsu HC. 1998. Association of downregulation of cyclin D1 and of overexpression of cyclin E with p53 mutation, high tumor grade and poor prognosis in hepatocellular carcinoma. J Hepatol 29:281289.

Petrocca F, Visone R, Onelli MR, Shah MH, Nicoloso MS, de Martino I, lliopoulos D, Pilozzi E, Liu CG, Negrini M, Cavazzini L, Volinia S, Alder H, Ruco LP, Baldassarre G, Croce CM, and Vecchione A. 2008. E2F1-regulated microRNAs impair TGFbeta-dependent cell-cycle arrest and apoptosis in gastric cancer. Cancer Cell 13:272286. 10.1016/j.ccr.2008.02.013

Ryan BM, O'Donovan N, and Duffy MJ. 2009. Survivin: a new target for anti-cancer therapy. Cancer Treat Rev 35:553562. 10.1016/j.ctrv.2009.05.003

Settles B. 2005. ABNER: an open source tool for automatically tagging genes, proteins and other entity names in text. Bioinformatics 21:3191-3192. 10.1093/bioinformatics/bti475

Shangguan H, Tan SY, and Zhang JR. 2015. Bioinformatics analysis of gene expression profiles in hepatocellular carcinoma. Eur Rev Med Pharmacol Sci 19:2054-2061.

Sharpless NE. 2005. INK4a/ARF: a multifunctional tumor suppressor locus. Mutat Res 576:22-38. 10.1016/j.mrfmmm.2004.08.021

Sherr CJ. 1996. Cancer cell cycles. Science 274:1672-1677.

Smith L, Tanabe LK, Ando RJ, Kuo CJ, Chung IF, Hsu CN, Lin YS, Klinger R, Friedrich CM, Ganchev K, Torii M, Liu H, Haddow B, Struble CA, Povinelli RJ, Vlachos A, Baumgartner WA, Jr., Hunter L, Carpenter B, Tsai RT, Dai HJ, Liu F, Chen Y, Sun C, Katrenko S, Adriaans P, Blaschke C, Torres R, Neves M, Nakov P, Divoli A, Mana-Lopez $\mathrm{M}$, Mata J, and Wilbur WJ. 2008. Overview of BioCreative II gene mention recognition. Genome Biol 9 Suppl 2:S2. 10.1186/gb-2008-9-s2-s2

Song B, Ai J, Kong X, Liu D, and Li J. 2013. Aberrant DNA Methylation of P16, MGMT, and hMLH1 Genes in Combination with MTHFR C677T Genetic Polymorphism in gastric cancer. Pak J Med Sci 29:1338-1343.

Torre LA, Bray F, Siegel RL, Ferlay J, Lortet-Tieulent J, and Jemal A. 2015. Global cancer statistics, 2012. CA Cancer J Clin 65:87-108. 10.3322/caac.21262

Wang J, Wen J, Yi R, Liu F, Zhou J, Liu G, Li Q, Yang Z, and Su X. 2015a. High selectivity of PI3Kbeta inhibitors in SETD2mutated renal clear cell carcinoma. J BUON 20:1267-1275.

Wang M, Wen TF, He LH, Li C, Zhu WJ, and Trishul NM. 2015b. A six-microRNA set as prognostic indicators for bile duct cancer. Int J Clin Exp Med 8:17261-17270.

Wang SN, Wang LT, Sun DP, Chai CY, Hsi E, Kuo HT, Yokoyama KK, and Hsu SH. 2016. Intestine-specific homeobox (ISX) upregulates E2F1 expression and related oncogenic activities in LIHC. Oncotarget. 10.18632/oncotarget.9228

Wang XW, Hussain SP, Huo TI, Wu CG, Forgues M, Hofseth L, Brechot C, and Harris CC. 2002. Molecular pathogenesis of human hepatocellular carcinoma. Toxicology 181-182:43-47.

Wei W, Wanjun L, Hui S, Dongyue C, Xinjun Y, and Jisheng Z. 2013. miR-203 inhibits proliferation of LIHC cells by targeting survivin. Cell Biochem Funct 31:82-85. 10.1002/cbf.2863

Xia Y, He Z, Liu B, Wang P, and Chen Y. 2015. Downregulation of Meg3 enhances cisplatin resistance of lung cancer cells through activation of the WNT/beta-catenin signaling pathway. Mol Med Rep 12:4530-4537. 
10.3892/mmr.2015.3897

Xu X, Wang X, Fu B, Meng L, and Lang B. 2015. Differentially expressed genes and microRNAs in bladder carcinoma cell line 5637 and T24 detected by RNA sequencing. Int J Clin Exp Pathol 8:12678-12687.

Yang Y, Zhu J, Gou H, Cao D, Jiang M, and Hou M. 2011. Clinical significance of Cox-2, Survivin and Bcl-2 expression in hepatocellular carcinoma (LIHC). Med Oncol 28:796-803. 10.1007/s12032-010-9519-y

Ye CP, Qiu CZ, Huang ZX, Su QC, Zhuang W, Wu RL, and Li XF. 2007. Relationship between survivin expression and recurrence, and prognosis in hepatocellular carcinoma. World J Gastroenterol 13:6264-6268.

Yim WW, Yetisgen M, Harris WP, and Kwan SW. 2016. Natural Language Processing in Oncology: A Review. JAMA Oncol 2:797-804. 10.1001/jamaoncol.2016.0213

Zender L, Spector MS, Xue W, Flemming P, Cordon-Cardo C, Silke J, Fan ST, Luk JM, Wigler M, Hannon GJ, Mu D, Lucito R, Powers S, and Lowe SW. 2006. Identification and validation of oncogenes in liver cancer using an integrative oncogenomic approach. Cell 125:1253-1267. 10.1016/j.cell.2006.05.030

Zhang E, Yin D, Han L, He X, Si X, Chen W, Xia R, Xu T, Gu D, De W, Guo R, Xu Z, and Chen J. 2015a. E2F1-induced upregulation of long noncoding RNA LINC00668 predicts a poor prognosis of gastric cancer and promotes cell proliferation through epigenetically silencing of CKIs. Oncotarget. 10.18632/oncotarget.6745

Zhang EL, Liang BY, Chen XP, and Huang ZY. 2015b. Severity of liver cirrhosis: a key role in the selection of surgical modality for Child-Pugh A hepatocellular carcinoma. World J Surg Oncol 13:148. 10.1186/s12957-015-05679

Zhang J, Chong CC, Chen GG, and Lai PB. 2015c. A Seven-microRNA Expression Signature Predicts Survival in Hepatocellular Carcinoma. PLoS One 10:e0128628. 10.1371/journal.pone.0128628

Zhang J, Fan D, Jian Z, Chen GG, and Lai PB. 2015d. Cancer Specific Long Noncoding RNAs Show Differential Expression Patterns and Competing Endogenous RNA Potential in Hepatocellular Carcinoma. PLoS One 10:e0141042. 10.1371/journal.pone.0141042

Zhang X, Hu S, Zhang X, Wang L, Zhang X, Yan B, Zhao J, Yang A, and Zhang R. 2014. MicroRNA-7 arrests cell cycle in G1 phase by directly targeting CCNE1 in human hepatocellular carcinoma cells. Biochem Biophys Res Commun 443:1078-1084. 10.1016/j.bbrc.2013.12.095

Zhang X, Tang W, Chen G, Ren F, Liang H, Dang Y, and Rong M. 2016. An Encapsulation of Gene Signatures for Hepatocellular Carcinoma, MicroRNA-132 Predicted Target Genes and the Corresponding Overlaps. PLoS One 11:e0159498. 10.1371/journal.pone.0159498

Zhang Y, Mao XY, Liu X, Song RR, Berney D, Lu YJ, and Ren G. 2015e. High frequency of the SDK1:AMACR fusion transcript in Chinese prostate cancer. Int J Clin Exp Med 8:15127-15136.

Zhong JH, Zhong QL, Li LQ, and Li H. 2014. Adjuvant and chemopreventive therapies for resectable hepatocellular carcinoma: a literature review. Tumour Biol 35:9459-9468. 10.1007/s13277-014-2443-6

Zhou Q, He Q, and Liang L. 2003. Expression of p27, cyclin E and cyclin A in hepatocellular carcinoma and its clinical significance. World J Gastroenterol 9:2450-2454.

Zhou YM, Zhang XF, Yu F, Liu XB, Wu LP, Li B, and Yang JM. 2014. Efficacy of surgical resection for pulmonary metastases from hepatocellular carcinoma. Med Sci Monit 20:1544-1549. 10.12659/MSM.890853 
Figure 1

General flow chart

Differentially expressed genes (DEGs) were generated by integrating data from The Cancer Genome Atlas (TCGA) and Natural Language Processing (NLP). Relevant signaling pathways of DEGs went through various analyses. The cancers-associated pathway ranked high in KEGG enrichment analysis was further investigated, and selected genes with top priority were evaluated and assessed in terms of their diagnostic and prognostic values.

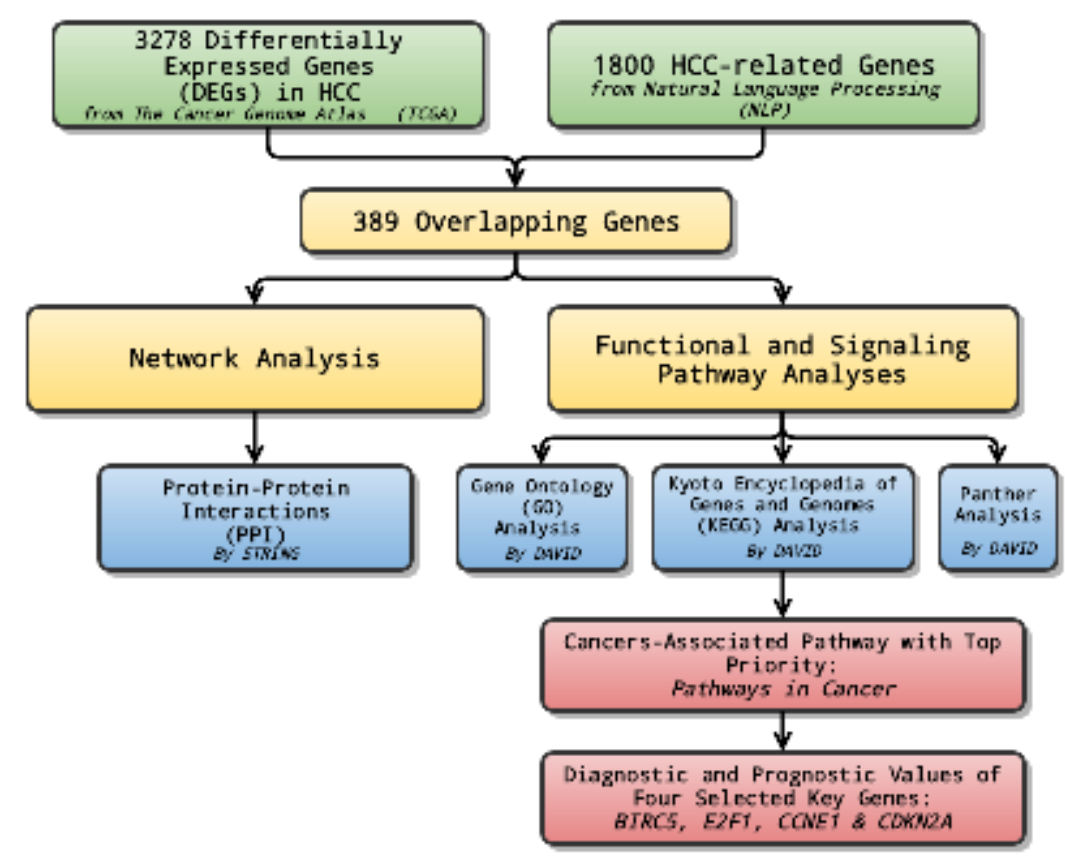


Figure 2

Interactions of the DEGs in hepatocellular carcinoma (HCC)

Interactions of 22 hub genes were illustrated by STRING online database (http://string-db.org) with the cut-off criterion of combined score $>0.7$. Network nodes represented proteins and edges stood for protein-protein associations.

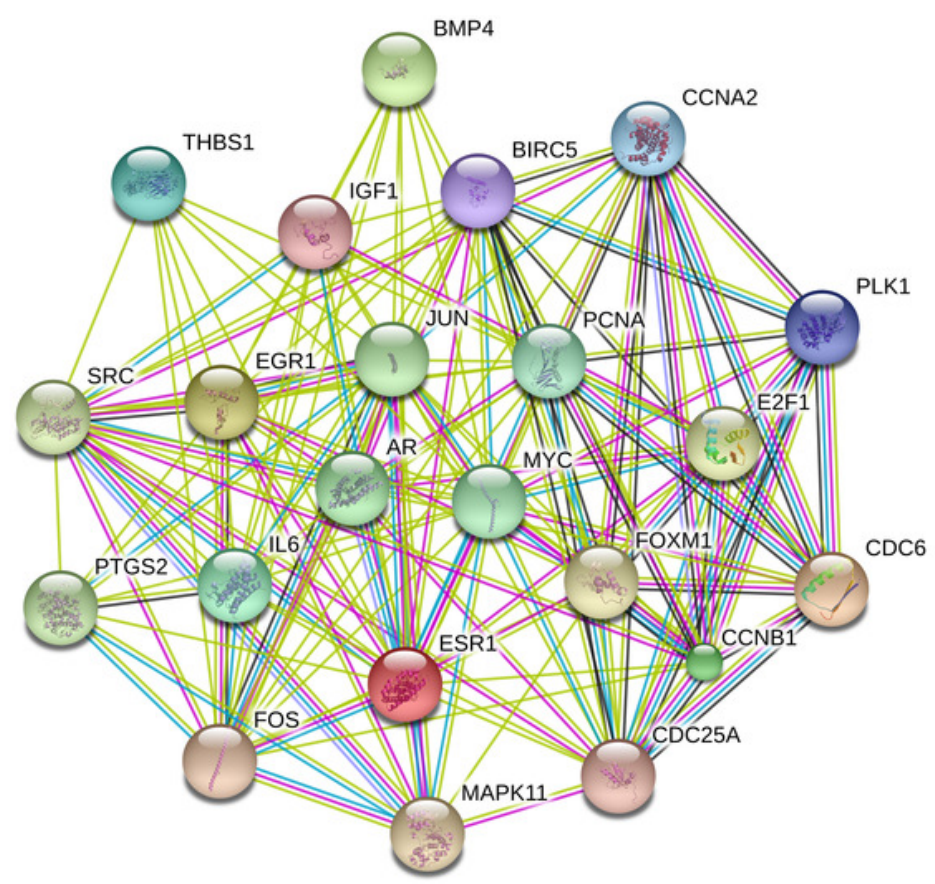


Figure 3

Directed acyclic graph (DAG) of pathways from the perspective of biological process (BP) in gene ontology $(\mathrm{GO})$ analysis

The circles represented different terms of biological processes. The relationships among terms were represented by arrows. A false discovery rate (FDR) of $10^{113}$ was selected for the current DAG with 44 nodes and 55 edges included. The darker the color appeared, the greater significance the term demonstrated.
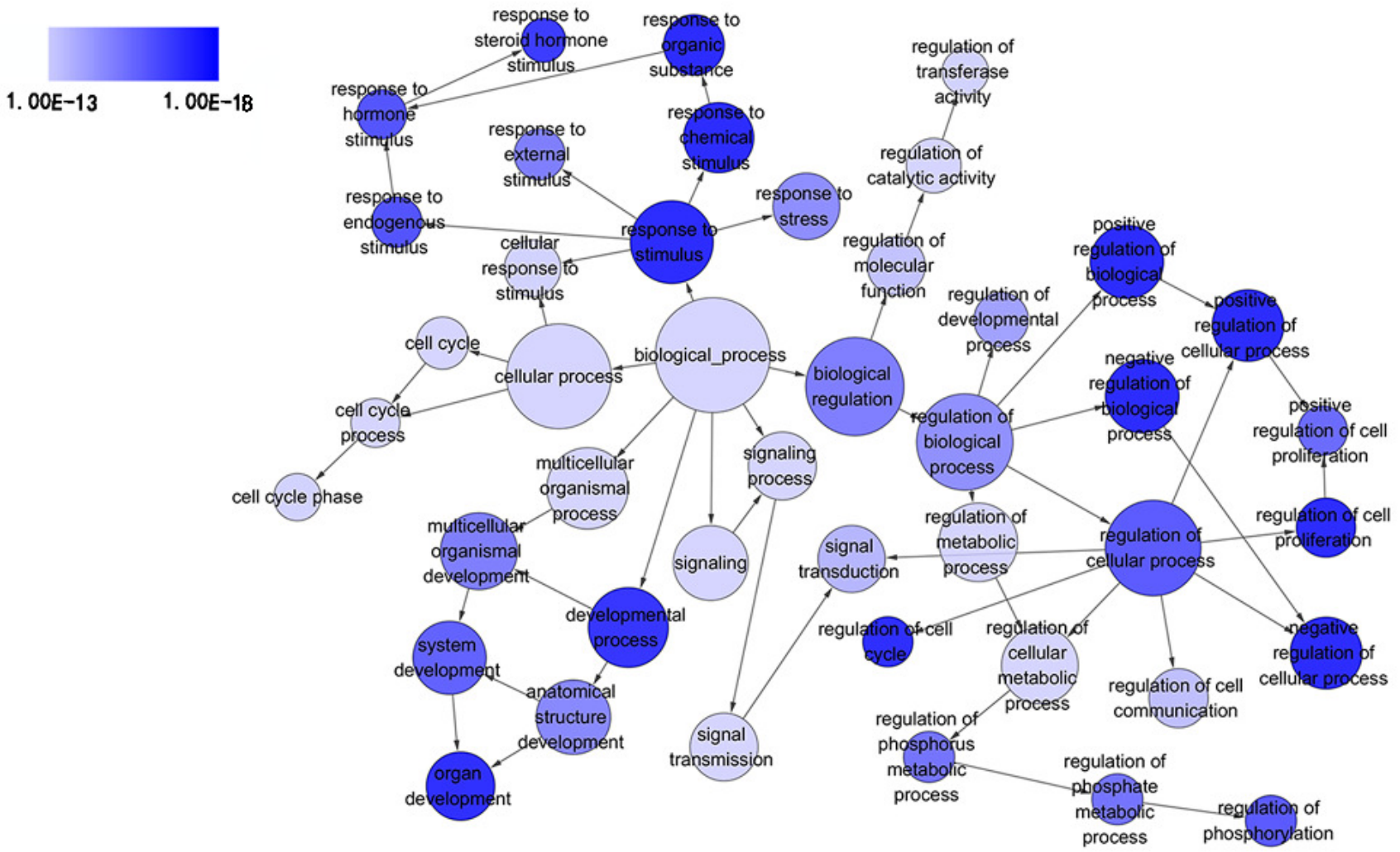


\section{Figure 4}

DAG of pathways from the perspective of cellular component (CC) in GO analysis

The circles represented different terms of cellular components. The relationships among terms were represented by arrows. A false discovery rate (FDR) of $10^{\square}$ was selected for the current DAG, which harbored 29 nodes and 45 edges. The color depth indicated the significance of the corresponding term.

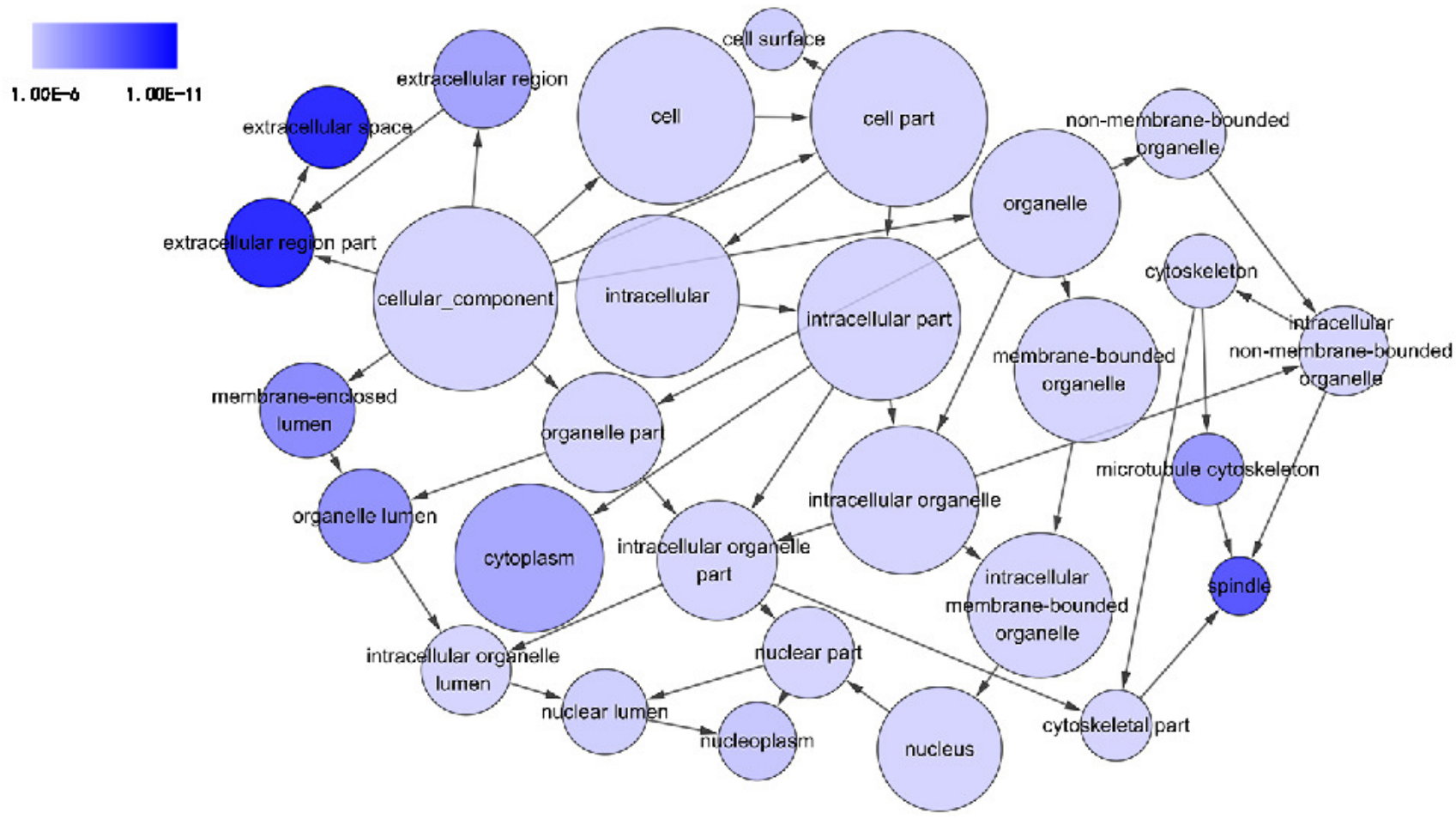




\section{Figure 5}

DAG of pathways from the perspective of molecular function (MF) in GO analysis

The circles represented different terms of molecular functions. The relationships among terms were represented by arrows. A false discovery rate (FDR) of $10^{\square}$ was selected for the current DAG, which held 32 nodes and 35 edges. The color depth indicated the significance of the corresponding term.

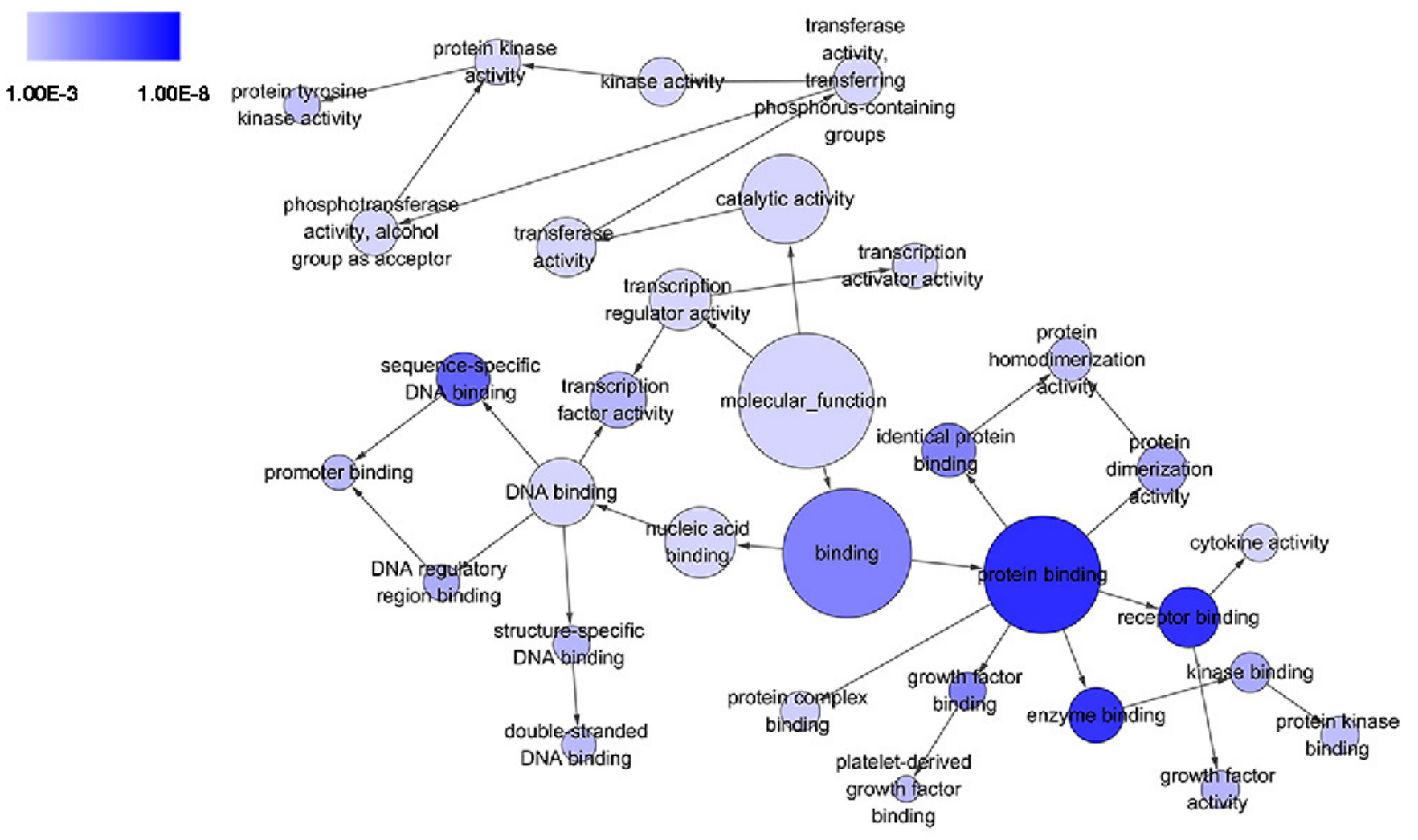




\section{Figure 6}

Gene Set Enrichment Analysis (GSEA) for DEGs in HCC

The 389 DEGs were displayed in form of a heat map achieved by GSEA (Human tissue compendium, Novartis) and MSigDB. 


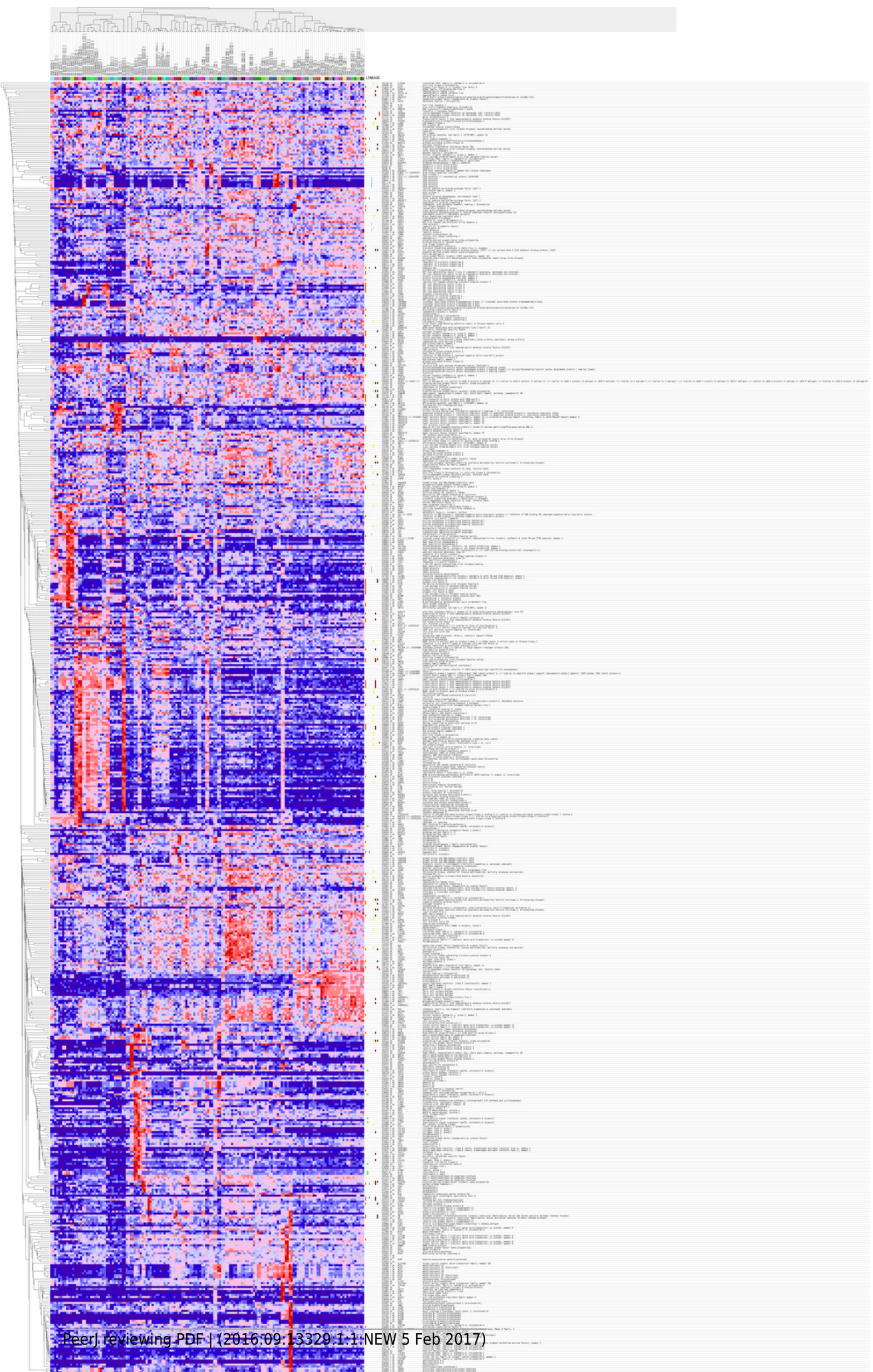




\section{Figure 7}

Top 15 Kyoto Encyclopedia of Genes and Genomes (KEGG) pathways of DEGs in HCC

The KEGG enrichment analysis was performed with 389 DEGs by ggplot2 package of $R$.

Pathways in cancer was the most significant pathway. The color tints indicated the P-values.

The size of the circle presented the number of selected genes in the pathway. Thirty genes were found to be enriched in pathways in cancer. Rich factor expressed the percentage of the ratio of DEGs in current study vs total genes in the pathway. 


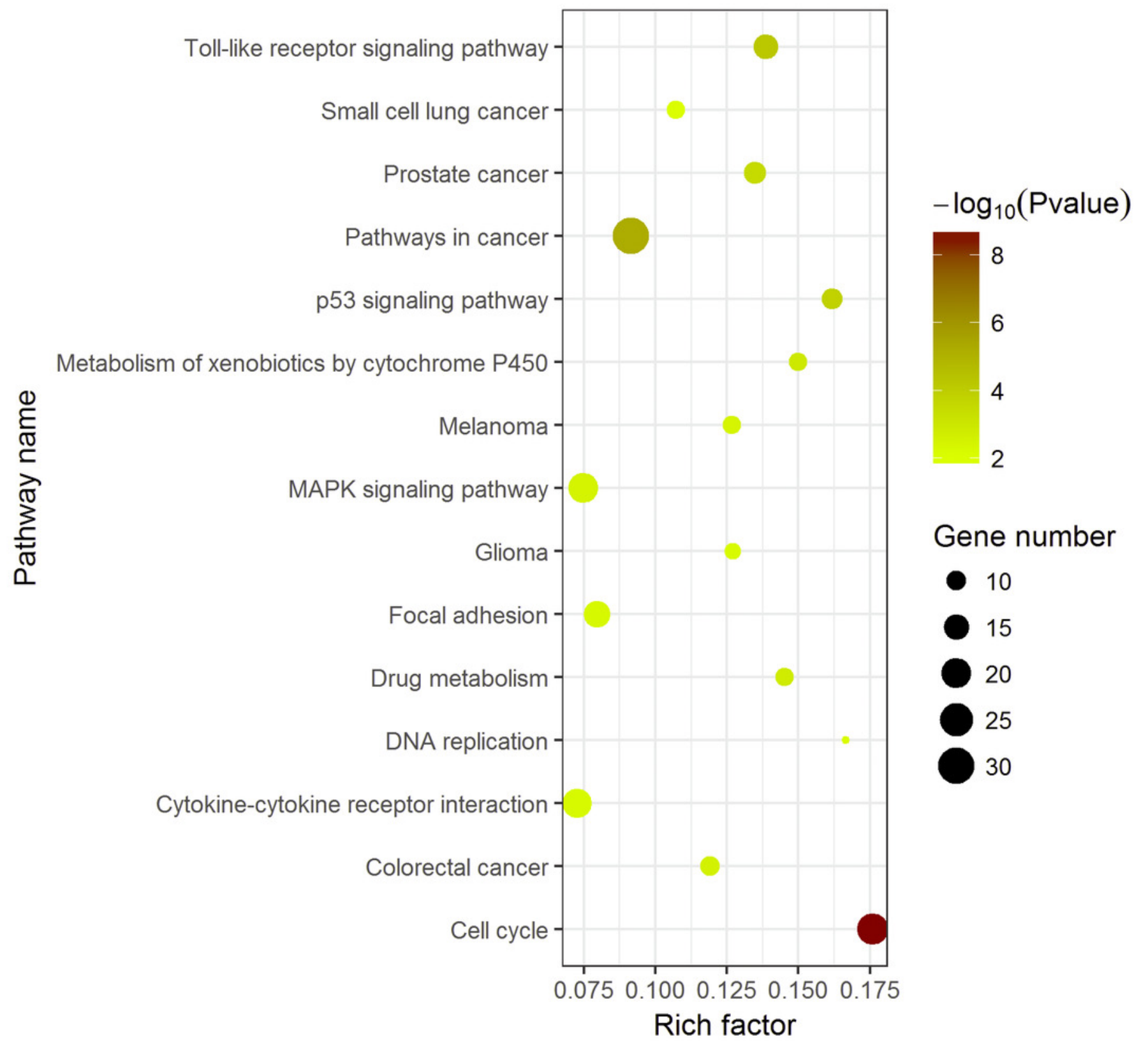




\section{Figure 8}

Location map of 30 genes from the current study in Pathways in Cancer

The figure was modified according to Pathway mapping tools

(http://www.genome.jp/kegg/mapper.html). Thirty genes from the current study were colored in green. Four significant genes (BIRC5, E2F1, CCNE1 and CDKN2A) providing the highest Area Under Curves (AUCs) were put in red, which located in different parts of the pathway. 


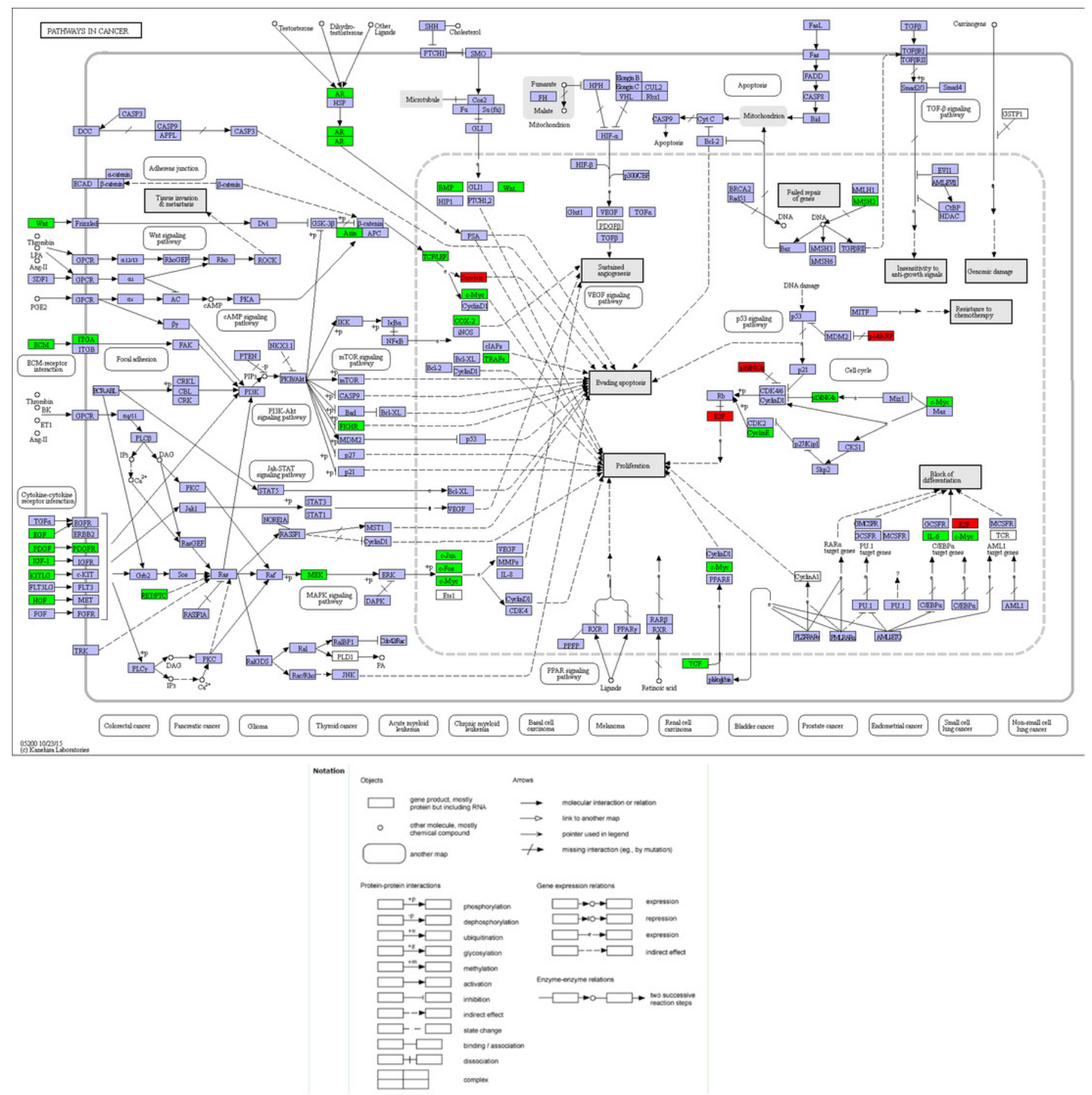


Figure 9

ROC curves of DEGs in HCC

There were 22 DEGs with AUCs greater than 0.8. A, B and C: ROC curves of the 12

upregulated genes in HCC; D and E: ROC curves of the 10 downregulated genes in HCC; F:

ROC curve of the detection pool of the four top-listing genes in HCC.

A

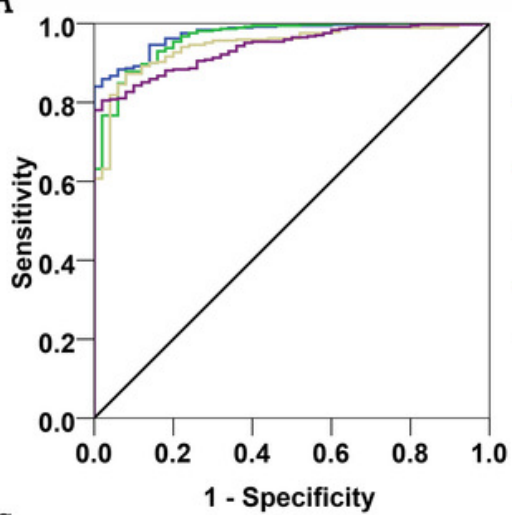

C

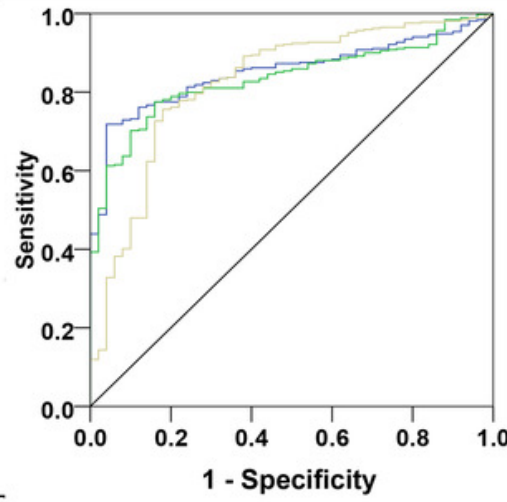

$\mathrm{E}$

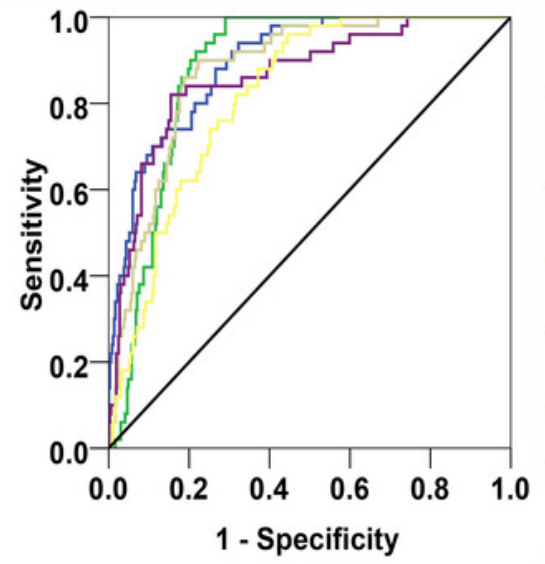

Source of the Curve

BIRC5 (AUC $=0.974$, $P<0.001)$

E2F1(AUC=0.964, $P<0.001)$

CCNE1 (AUC $=0.945$, $\mathrm{P}<0.001)$

CDKN2A(AUC $=0$.

939, $\mathrm{P}<0.001)$

-Reference Line

Source of the Curve PDGFA(AUC $=0.851$, $P<0.001)$

LEF1 $(A U C=0.834$,

$P<0.001)$

CDKN2B(AUC $=0.824$, $P<0.001)$

- Reference Line

Source of the Curve

-IGF1 (AUC= 0.895,

$P<0.001)$

PDGFRA(AUC $=0.880$, $P<0.001)$

RET(AUC=0.876, $P<0$.

001)

JUN(AUC=0.863, $\mathrm{P}<0$.

001)

$A R(A U C=0.818, P<0$.

001)

- Reference Line
B

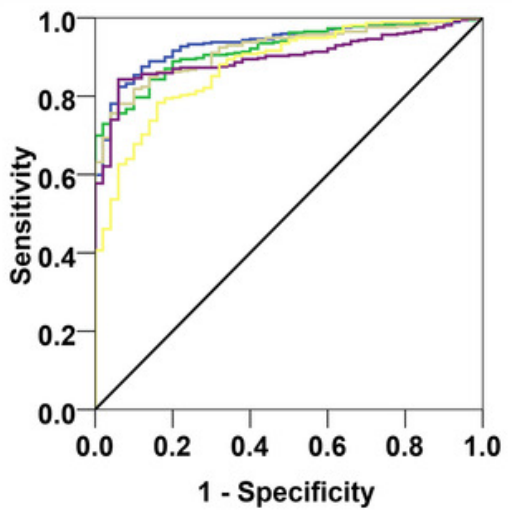

D

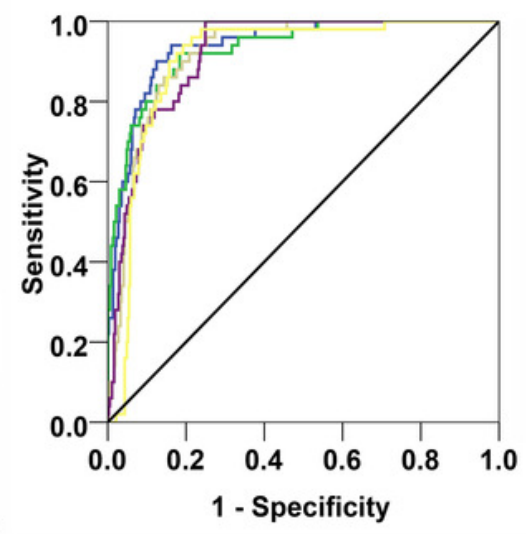

F

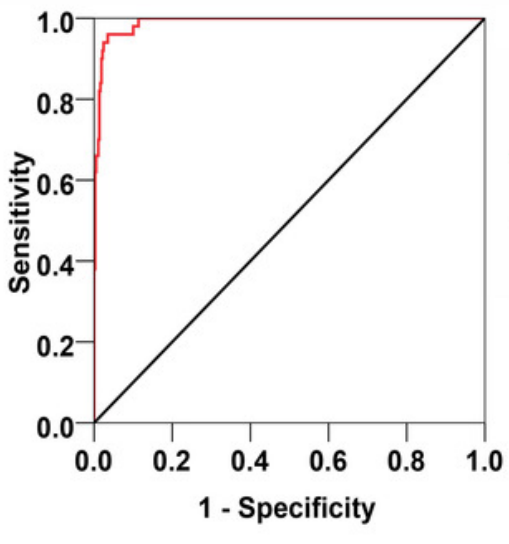

Source of the Curve

$-\mathrm{MSH} 2(\mathrm{AUC}=0.934$

$P<0.001)$

-TRAF2(AUC $=0.921$ )

LAMA4(AUC $=0.918$

$P<0.001)$

CCNE2(AUC $=0.899$

$P<0.001)$

ITGA6(AUC $=0.878$

$P<0.001)$

-Reference Line

Source of the Curve

_FOS(AUC $=0.948$,

$P<0.001)$

MAP2K1 $(A \cup C=0$.

931, $\mathrm{P}<0.001)$

PTGS2(AUC $=0.920$,

$P<0.001)$

FOXO1 (AUC $=0.919$,

$P<0.001)$

HGF(AUC $=0.905$,

$P<0.001)$

-Reference Line

Source of the Curve

BIRC5,E2F1, CCNE1, CDKN2A (AUC $=0.990$ $\mathrm{P}<0.001$ )

-Reference Line 


\section{Figure 10}

Expression levels of the top four DEGs in HCC from TCGA data

The figures illustrated the expression levels of the top four DEGs in HCC tissues from TCGA data as compared to para-HCC non-cancerous tissues. A: BIRC5; B: E2F1; C: CCNE1 and D: CDKN2A. 
A

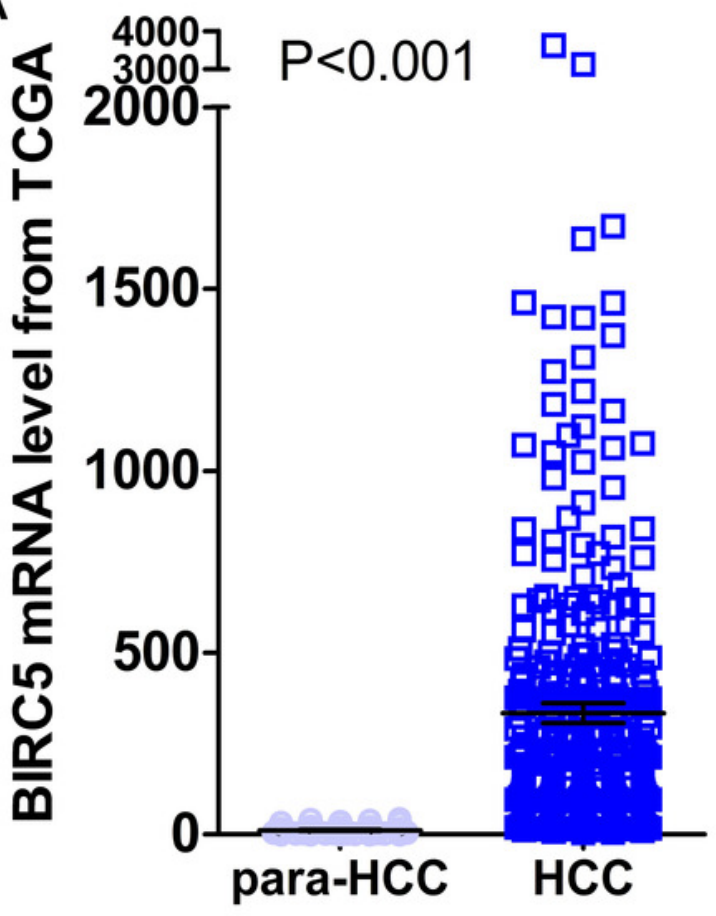

C

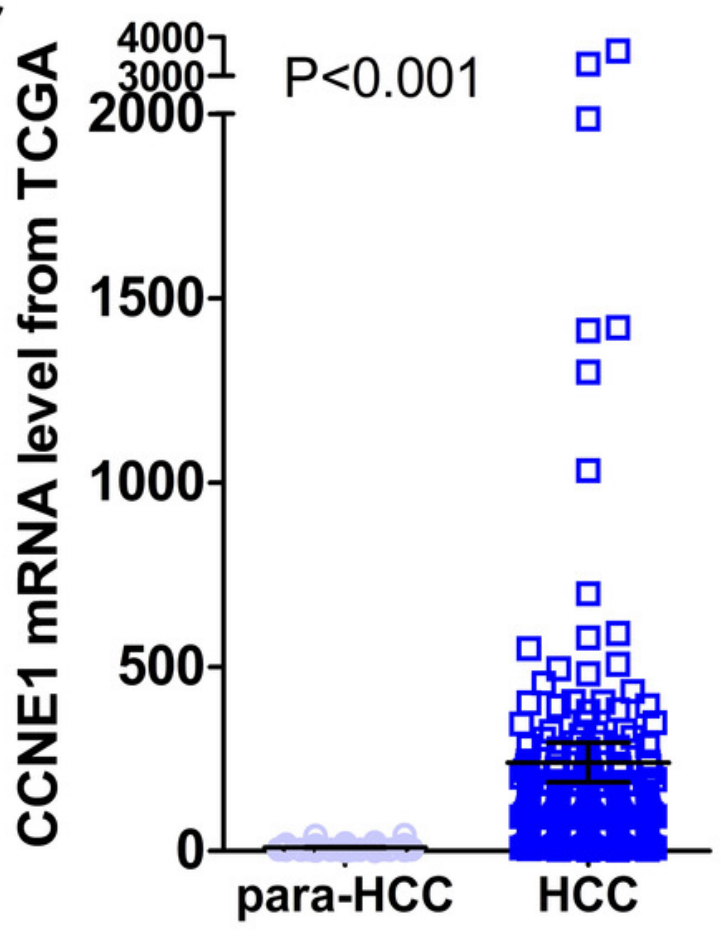

B

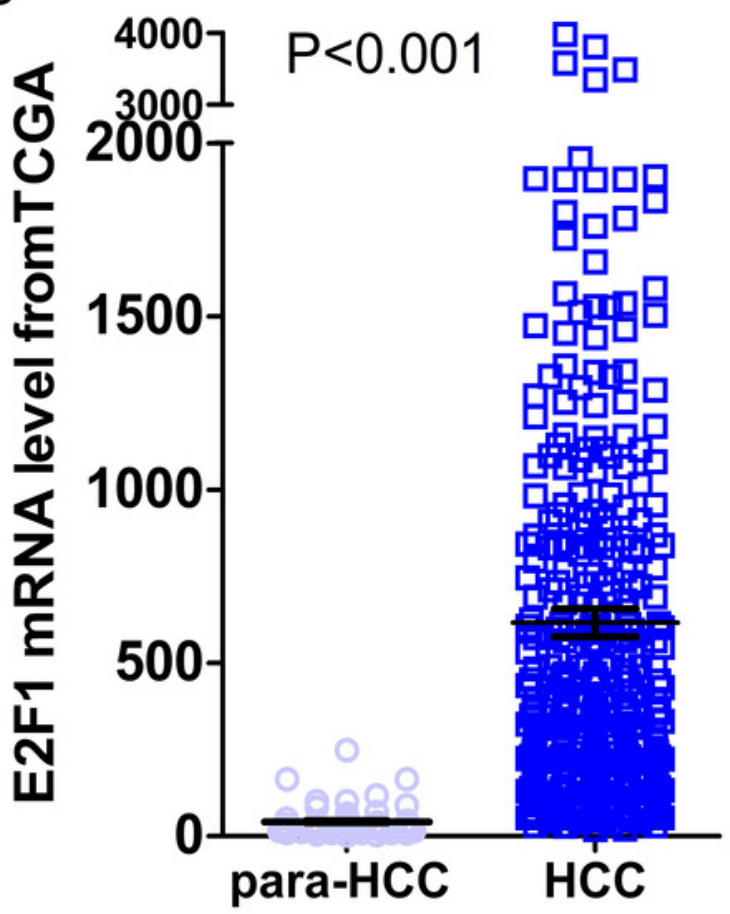

D

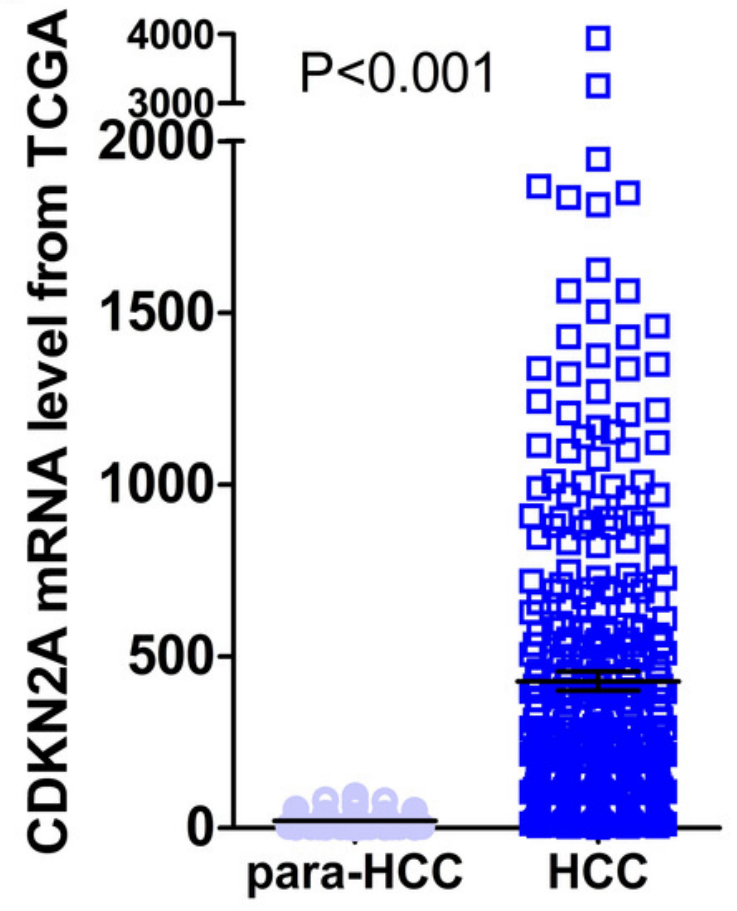


Figure 11

Associations of the top four DEGs with overall survival (OS) of 369 patients from TCGA data

Kaplan-Meier survival analysis of OS based on expression status provided the associations of the top four DEGs with overall survival (OS) of 369 patients from TCGA data A: BIRC5 $(* P=0.021$, log-rank); B: CCNE1 (*P=0.027); C: CDKN2A $(P=0.066) ; D:$ E2F1 $(P=0.088)$.

A

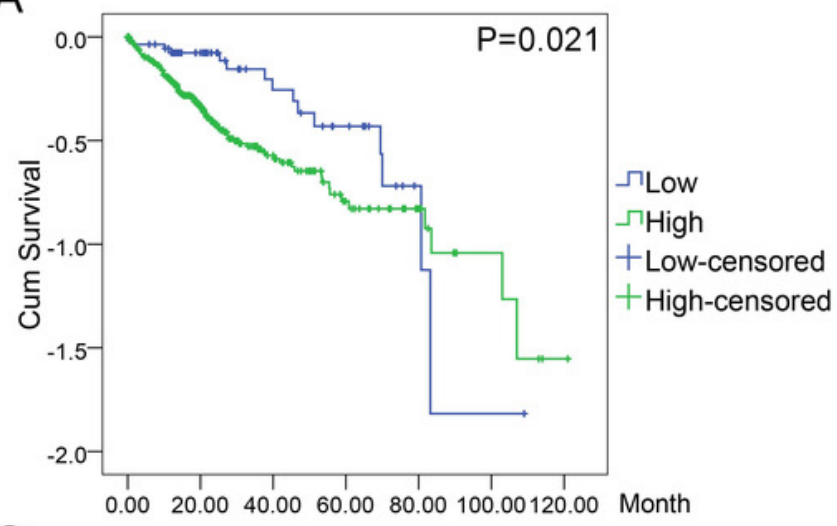

C

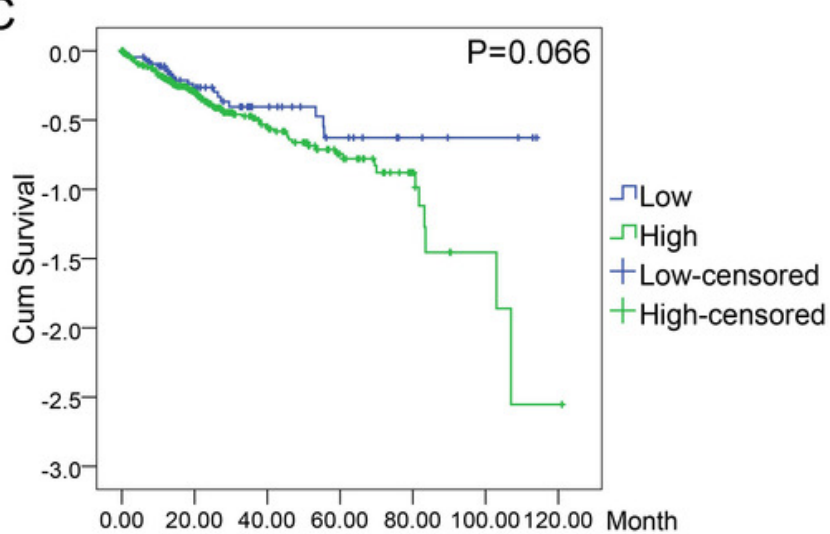

B

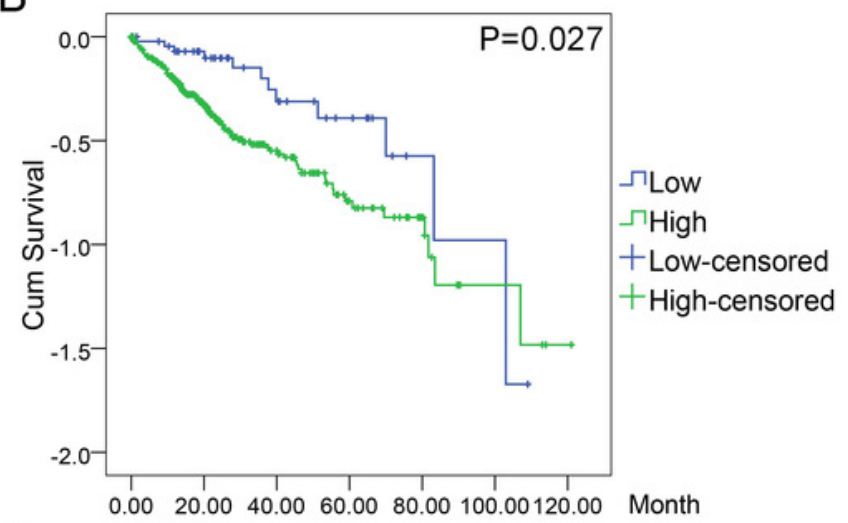

D

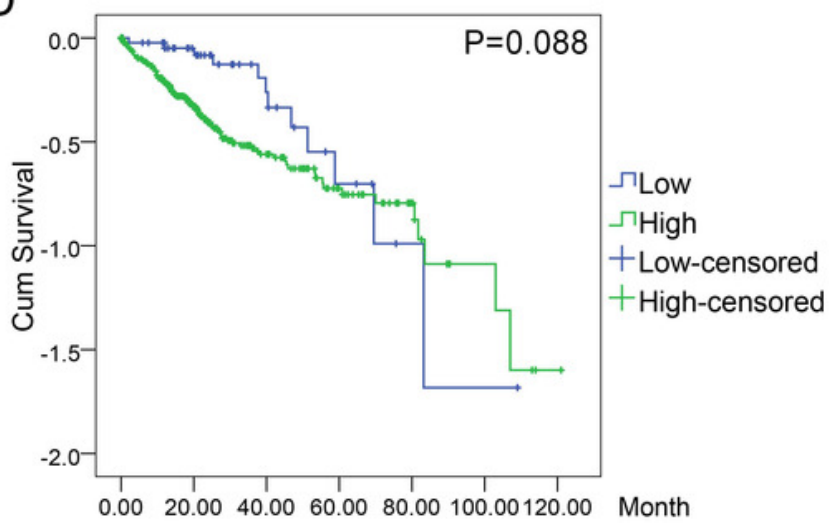

